Classification

Physics Abstracts

$07.50-41.10 \mathrm{~F}$

\title{
Etude des perturbations conduites et rayonnées dans une cellule de commutation
}

\author{
F. Costa $\left({ }^{1}\right)$, F. Forest $\left({ }^{1}\right)$, A. Puzo $\left({ }^{2}\right)$ et G. Rojat $\left({ }^{2}\right)$ \\ (1) LESIR-URA n 1375, ENS de Cachan, 61 Avenue du Président Wilson, 94230 Cachan, \\ France \\ (2) CEGELY-URA n 829, Ecole Centrale de Lyon, 36 Avenue G. de Collongue, 69131 Ecully \\ Cedex, France
}

(Reçu le 10 juin 1993, révisé et ac'epté le 8 septembre 1993)

\begin{abstract}
Résumé. - Les principes de commutation employés en conversion statique, l'évolution des performances statiques et dynamiques des composants, contribuent à faire des dispositıfs de conversion statique de puissants générateurs de perturbations conduites et rayonnées. Nous nous sommes attachés à étudier les mécanismes de génération et de couplage des perturbations, tant en mode conduit que rayonné dans des structures à une seule cellule de commutation et fonctionnant selon les trois principaux modes de commutation : commutation forcée, à zéro de courant (ZCS), et à zéro de tension (ZVS). Après la mise en évidence de la problématique de pollution électromagnétique dans les structures et leur métrologie, nous décrivons l"environnement expérimental étudié. Nous analysons ensuite les principaux mécanismes produisant les perturbations au sein d'une cellule de commutation en introduisant un certain nombre de composants parasites. Les modèles sont simulés et confrontés aux résultats expérimentaux. Nous décrivons alors une méthode, validée expérımentalement et permettant de calculer les intensités des champs $E$ et $H$ proches émis. Enfin. nous présentons de façon synthétique les résultats observés selon les régimes de fonctionnement de la cellule de commutation et les contraintes électriques et dynamiques qu'elle subit. Nous avons, pour ce faire, développé une méthode originale de quantification des signaux perturbateurs. Les résultats obtenus doivent permettre d'intégrer les problèmes de pollution électromagnétique au stade de la conception d'un dispositif.
\end{abstract}

Abstract. - The principles used in static conversion and the rise of the performances of the new switching devices contribue to increase the level of electromagnetic noises emitted by electronic converters. We have studied the way how these perturbations are created and coupled through their environment in conducted and radiated mode by a switching cell. This one can work in hard switching, zero current or voltage switching modes. We first outline the general problems of electromagnetic pollution and their metrology in converters. Then we describe the experimental environment. We analyse the mechanisms of generation of parasitic signals in a switching cell related to the electrical constraints and its switching mode. The simulated results, issued of the analytical models obtained, are confronted with the experimental ones. Then we show a method to calculate analytically the $E$ and $H$ near fields. It has been confirmed by experimental results. At last, we present, in a synthetic manner, the main results obtained, relative to the switching mode and the electrical constraints, using a new characterizing method. Theses results will allow the designer to incorporate the electromagnetic considerations in the conception of a converter. 


\section{Présentation.}

1. 1 PhÉNOMÈnes GÉnÉRAuX [1, 2, 12-14]. - Aujourd'hui, la conversion d'énergie utilise quasi-exclusivement des principes de commutation, favorisés par les performances élevées des composants de commutation, tant en limites statiques que dynamiques. L'émergence des composants à grille isolée, MOSFET et IGBT, permet d'envisager des fréquences de découpage de l'ordre de plusieurs $\mathrm{MHz}$ avec des contraintes statiques de l'ordre de $100 \mathrm{~V}$ et $10 \mathrm{~A}$ pour les premiers et $10 \mathrm{kHz}-1200 \mathrm{~V}-200 \mathrm{~A}$ pour les seconds. La conséquence de ce principe est l'existence inhérente de variations rapides de tension et de courant dans la structure de conversion, d'autant plus élevées que les contraintes statiques le sont. Par ailleurs les différents modes de commutation (dure, à zéro de courant, à zéro de tension) occasionnent un comportement dynamique spécifique de l'interrupteur.

Ces phénomènes sont sources de perturbations électromagnétiques conduites en mode commun et différentiel, ainsi que rayonnées en champs électriques et magnétiques proches et lointains.

Nous analysons dans ce travail le rôle central joué par l'interrupteur et de son mode de commutation dans la génération des perturbations.

\subsection{INSTRUMENTATION EN COMPATIBILITÉ ÉLECTROMAGNÉTIQUE.}

1.2.1 Mesures des grandeurs conduites [3, 4]. - Les courants parasites conduits sont caractérisés en mode commun et différentiel. Le dispositif réalisant cette mesure est le RSIL (Réseau Stabilisé d'Impédance de Ligne). Il doit être inséré dans un environnement assurant une bonne répétitivité de mesure (Fig. 1).

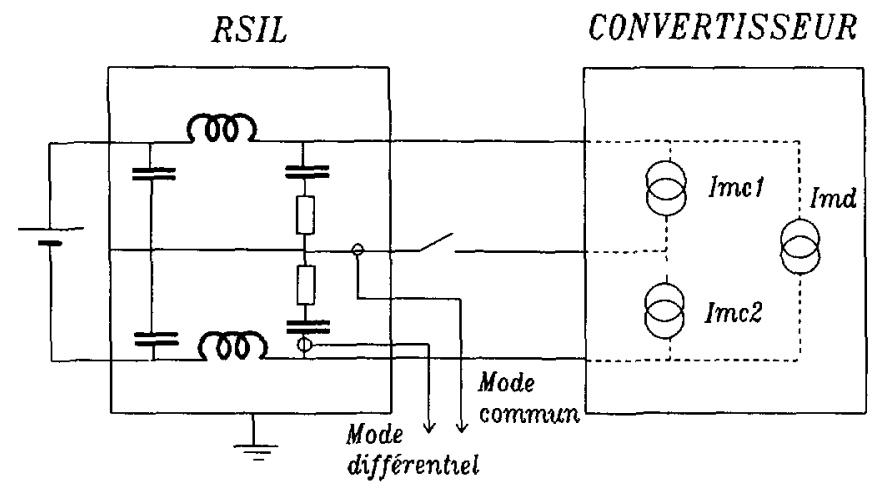

Fig. 1. - Dispositif de mesure des courants conduits.

[Conducted current measuring device.]

Le RSIL s'apparente à un filtre passe-haut vis-à-vis des courants parasites HF délivrés par le convertisseur, ceux-ci sont mesurés aux bornes d'une résistance normalisée de 50 Ohms. Il constitue un filtre passe-bas vis-à-vis de perturbations en provenance du réseau où il est inséré. Les normes le caractérisent dans une plage de fréquence de $100 \mathrm{kHz}$ à $30 \mathrm{MHz}$.

Le but de ce travail consiste à étudier les perturbations HF à la source pour une cellule de commutation non réversible. Les régimes parasites peuvent atteindre $50 \mathrm{MHz}$ aussi a-t-il été nécessaire de réaliser un RSIL large bande connecté directement aux bornes du convertisseur. Les mesures sont assurées par des sondes de courant à très large bande passante $(9 \mathrm{kHz}-$ 
$200 \mathrm{MHz}$ ) et permettent la séparation des modes communs et différentiels en ouvrant la connexion au châssis du convertisseur.

1.2.2 Mesure des grandeurs rayonnées. — L étude des mécanismes de perturbations rayonnées nécessite l'accès aux grandeurs des champs électriques et magnétiques autour du convertisseur statique. Les contraintes fréquencielles et directionnelles des sources d'émission imposent l'utilisation d'antennes. Il est primordial de connaître leur fonction de transfert avec précision de manière à pouvoir reconstituer le signal temporel.

La détermination expérimentale du rayonnement d'un convertisseur nous impose des mesures en champ proche de manière à caractériser avec précision l'origine et la forme des perturbations émises. Le choix des antennes a donc été considérablement restreint pour chaque forme d'émission. Les antennes doivent avoir des dimensions faibles par rapport à la source et d'autant plus réduites que celle-ci est proche. Les mesures de rayonnement en champ magnétique $H$ sont effectuées grâce à des antennes boucles passives couvrant une bande de fréquence de $100 \mathrm{kHz}$ à $100 \mathrm{MHz}$, leur structure étant composée d'un anneau conducteur de diamètre 1,3 et $6 \mathrm{~cm}$, relié à un câble coaxial de $50 \Omega$. La mesure du champ électrique $E$ nécessite des antennes de dimensions faibles. de grand gain, de bande de fréquence similaire aux précédentes et si possible directives de manière à mieux localiser les sources perturbatrices. L'absence sur le marché de tels composants réunissant ces critères nous a conduit à développer et à réaliser des antennes monopoles actives.

1.2.3 Récepteurs de mesure. - Deux modes de représentation des signaux captés par les différentes sondes (RSIL, sondes de tension ou de courant, antennes) sont utilisés:

- analyseur de spectres dans le domaine fréquentiel : il devra travailler en bande étroite, le filtre de résolution doit être inférieur à la fréquence de découpage la plus basse du convertisseur. La détection crête est bien adaptée à la mesure CEM et on cherchera à optimiser le rapport signal/bruit par moyennage des relevés ;

- analyseur de signaux dans le domaine temporel : les contraintes dynamiques des signaux observés imposent une bande passante et une fréquence d'échantillonnage de l'ordre de $100 \mathrm{MHz}$. L'observation simultanée des phénomènes à l'échelle de la période de découpage et des transitoires de commutation impose de plus une profondeur de mémoire d'acquisition importante (jusqu'à $64 \mathrm{Ko}$ ). Ce type d'instrument permet d'effectuer des opérations de traitement du signal ou de mesure très utiles dans l'analyse des mécanismes de perturbation et de couplage [4].

1.3 SuPPORT DE L'ÉTUdE [3, 4]. - L'environnement de mesure est représenté figure 2. Le convertisseur, constitué d'une cellule de commutation, est réalisé sur un circuit imprimé, les différentes mailles sont coplanaires et séparées permettant le découplage des champs proches, toutes les grandeurs électriques (courant, tension) sont facilement accessibles. Le dissipateur, sur lequel sont fixés les composants actifs, constitue le châssis et supporte le circuit imprimé.

Pour éviter les perturbations véhiculées par le réseau, l’alimentation du convertisseur et de sa commande sont réalisées par batterie, la commande est transmise par fibre optique. Les convertisseurs évalués sont :

- un hacheur non isolé à commutation forcée assistée (Fig. 3a) ;

- un hacheur non isolé en mode quasi-résonnant (ZCS) (Fig. 3b) ;

- un onduleur à résonance série (ZVS) (Fig. 3c).

Ils fonctionnent dans la gamme $1 \mathrm{~kW}-100 \mathrm{kHz}$, sous une tension d'alimentation de $100 \mathrm{~V}$. Les interrupteurs sont des transistors MOS de calibre $250 \mathrm{~V}-20 \mathrm{~A}$. Les structures de hacheur sont construites autour d'une cellule mono-interrupteur de commutation. 


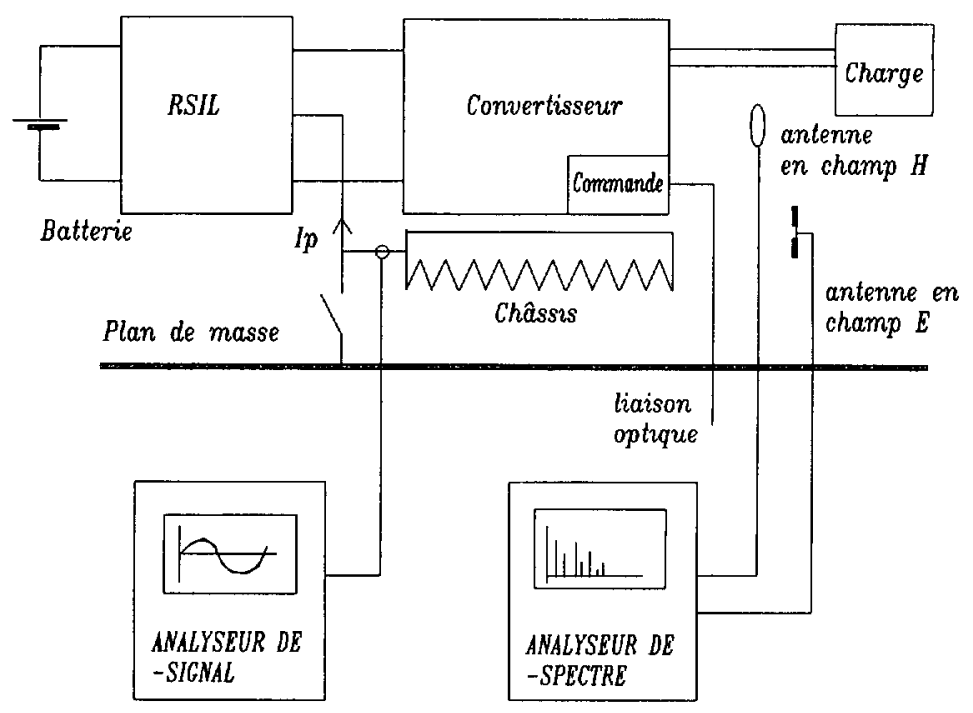

Fig. 2. - Environnement expérimental.

[Experimental environment.]

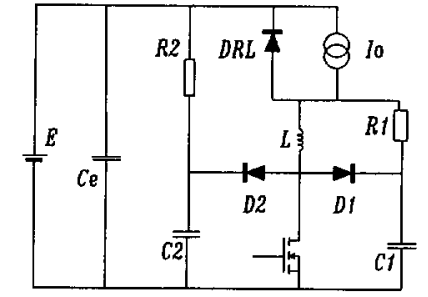

HACHEUR A COMMUTATION FORCEE

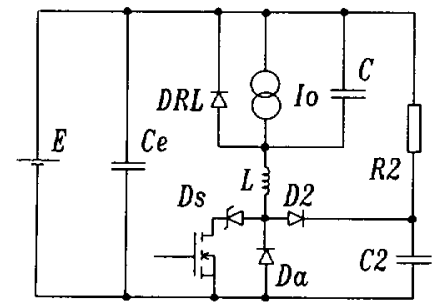

HACHEUR QUASI-RESONNANT (mode ZCS)

a)

b)

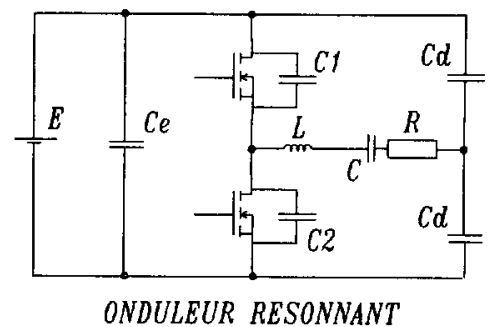

(mode ZVS)

c)

Fig. 3. - Structures étudiées.

[Studied structures.] 


\section{La CEM dans un convertisseur statique.}

Les perturbations conduites et rayonnées par un convertisseur statique ont une origine commune, déterminée par son fonctionnement électrique et sa disposition matérielle. A la structure électrique initiale, on ajoute des composants parasites localisés. Ceci permet de déterminer par simulation numérique les formes d'ondes du convertisseur et les différents courants conduits. En faisant intervenir les aspects géométriques, on obtient par calcul les différents champs électromagnétiques. Ces résultats sont ensuite validés par la mesure.

2.1 ORIGINE DES PERTURBATIONS DANS UN CONVERTISSEUR. — Le schéma électrique du convertisseur ne suffit pas à expliquer les formes d'ondes réellement observées et a fortiori les perturbations EM qui en résultent.

En effet, le câblage et la topologie vont introduire des éléments parasites et des couplages. Il est donc nécessaire d'introduire deux types de composants parasites dans le schéma du convertisseur (Fig. 4).

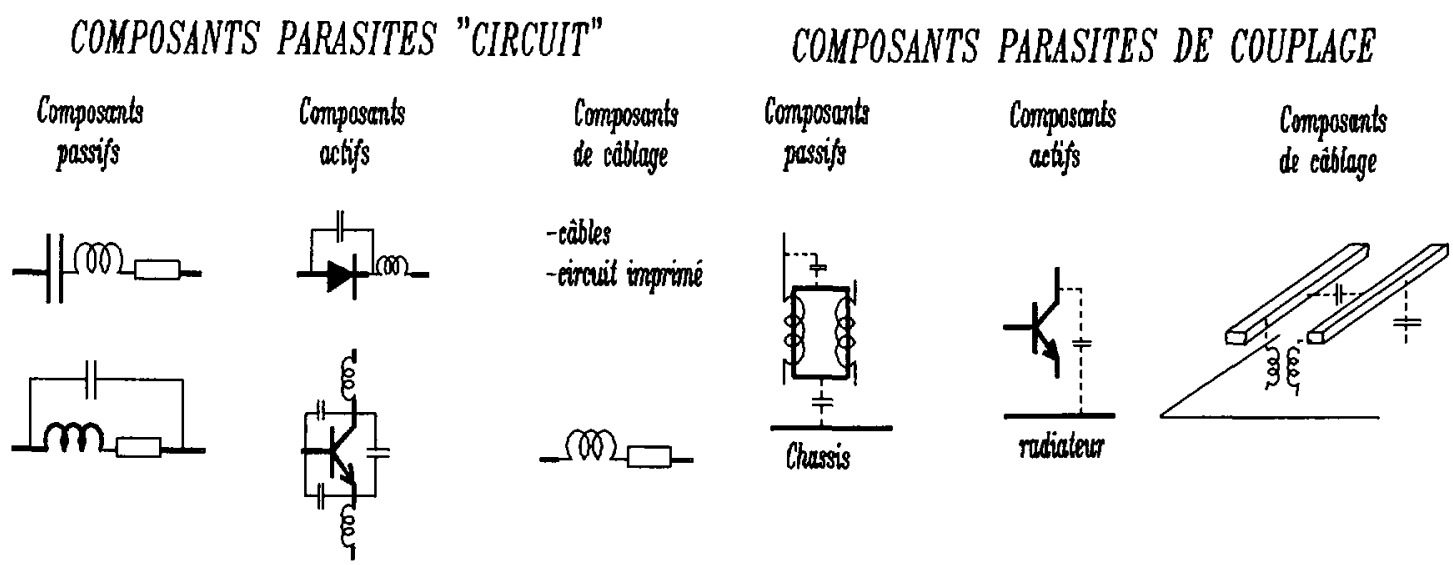

Fig. 4. - Les différents composants parasites.

[Different parasitic components.]

- Les composants parasites de type "circuit": inductances de câblage, capacités de semiconducteur, imperfections inductives ou capacitives des composants passifs. Ils modifient les formes d'onde théoriques du convertisseur et créent les régimes parasites souvent oscillatoires à fréquence élevée. Ils se superposent aux formes d'ondes théoriques et interviennent directement sur le courant absorbé en mode différentiel.

- Les composants parasites de couplage : dans les structures étudiées, ils sont constitués essentiellement par les capacités boîtier-radiateur des semi-conducteurs ou de câblage vis-à-vis du châssis. Ces éléments sont répartis mais leur rôle est prépondérant vis-à-vis des équipotentielles à forts $\mathrm{d} V / \mathrm{d} t$ dans la structure.

2.2 Calcul des Composants parasites $[5,6]$. - A partir des résultats d'électrostatique, de magnétostatique et d'hyperfréquence, nous avons déterminé les expressions analytiques des valeurs linéiques des inductances et capacités par rapport à un plan de masse pour des conducteurs de géométrie simple (rectangulaire et cylindrique) et supposés rectilignes. Ces topologies modélisent assez bien le circuit imprimé et le câblage du convertisseur au-dessus de 
son châssis. Les résultats ont été confirmés expérimentalement et d'autre part le logiciel Fissure [7] a permis de valider ces expressions. Les différents résultats analytiques sont donnés sur le tableau I. L'introduction d'éléments parasites localisés dans le schéma de la structure doit se limiter aux endroits où ils auront une action significative pour limiter la complexité du schéma et son analyse. La figure 5 donne le modèle obtenu pour le hacheur à commutation forcée de la figure $3 a$.

Tableau I. - Expressions analytiques des composants parasites.

[Analytical expressions of parasitic components.]

\begin{tabular}{|c|c|c|c|}
\hline$/$ & & \\
\hline$L p=\frac{\mu_{0}}{2 \pi} \cdot \ln \left(\frac{4 h}{d}\right)$ & $L p=\frac{\mu_{0}}{\pi} \cdot \ln \left(\frac{2 D}{d}\right)$ & $L p=\frac{\mu_{0}}{2 \pi} \cdot \ln \left(\frac{8 h}{L}+\frac{L}{4 h}\right)$ & $L p=\frac{\mu_{o}}{\pi} \cdot \ln \left(\frac{\pi d}{L}\right)$ \\
$C p=\frac{2 \pi \cdot \varepsilon_{0}}{\ln \left(\frac{4 h}{d}\right)}$ & $C p=\frac{\pi \cdot \varepsilon_{0}}{\ln \left(\frac{2 D}{d}\right)}$ & $C p=\frac{2 \pi \cdot \varepsilon_{0}}{\ln \left(\frac{8 h}{L}+\frac{L}{4 h}\right)}$ & $C p=\frac{\pi \cdot \varepsilon_{0}}{\ln \left(\frac{\pi d}{L}\right)}$ \\
\hline
\end{tabular}

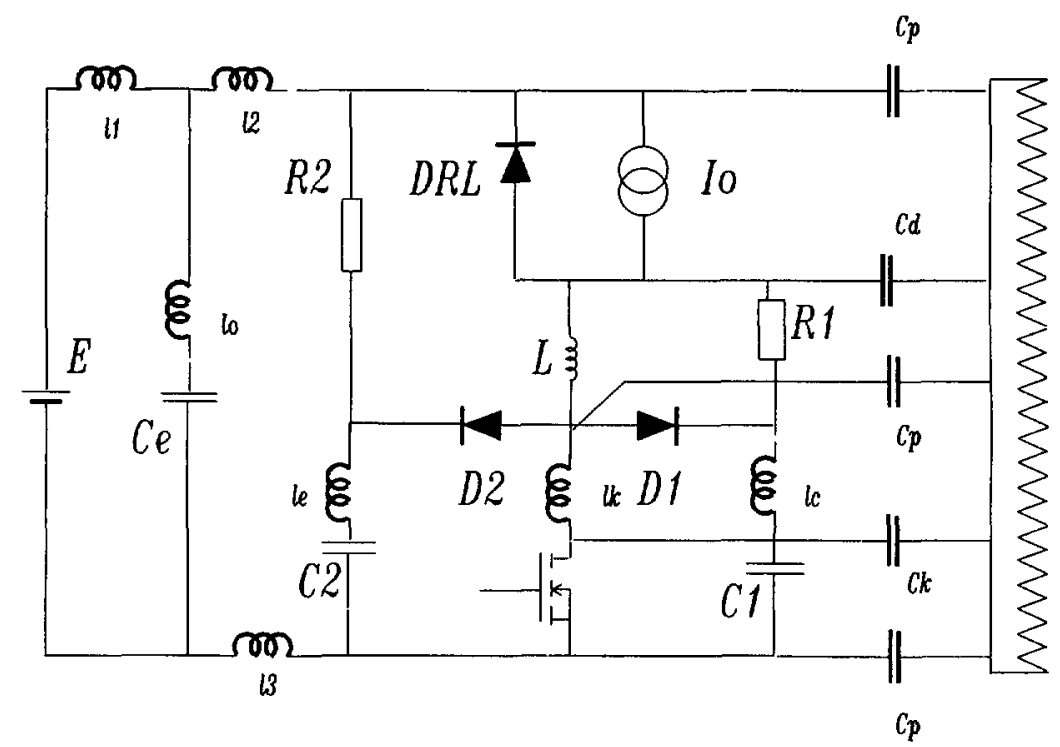

Fig. 5. - Modèle de la cellule de commutation avec les composants parasites.

[Model of the switching cell with parasitic components.] 
Le tableau II, résume les valeurs des différents éléments. Les hypothèses faites concernant les expressions analytiques précédentes ne correspondent pas exactement à la disposition géométrique du câblage, aussi les résultats présentés montrent quelques écarts entre les valeurs calculées (ou modélisées) et les valeurs expérimentalement mesurées surtout sur les faibles valeurs. Toutefois les résultats obtenus restent significatifs, les différences relatives étant de l'ordre de $20 \%$.

Tableau II. - Comparaison des valeurs calculées, mesurées et simulées.

[Measured results compared to calculated ones.]

\begin{tabular}{|c|c|c|c|}
\hline $\begin{array}{c}\text { Inductances parasites } \\
(\mathrm{nH})\end{array}$ & Calcul analytique & Mesures & $\begin{array}{c}\text { Modélisation } \\
\text { (FISSURE) }\end{array}$ \\
\hline $\mathrm{L2}$ & 59,4 & 45 & 79 \\
\hline $\mathrm{Lk}$ & 53,5 & 41,6 & 58 \\
\hline $\mathrm{Lc}$ & 26,7 & 20,6 & 36 \\
\hline $\mathrm{Le}$ & 70,8 & 62,5 & 80 \\
\hline $\mathrm{L} 3$ & 59,4 & 45 & 79 \\
\hline $\mathrm{L} 0$ & 79,2 & 54 & 100 \\
\hline & & & Modélisation \\
\hline Capacités parasites & Calcul & Mesure & 1,8 \\
\hline (pF) & 3 & 8 & 14,7 \\
\hline $\mathrm{Cp}$ & 20 & 23 & 3 \\
\hline $\mathrm{Cd}$ & 5 & 8 & 19,6 \\
\hline $\mathrm{Cp}$ & 25 & 29 & 1,8 \\
\hline $\mathrm{Ck}$ & 3 & 9 & \\
\hline
\end{tabular}

2.3 MÉCANISMES ÉLECTRIQUES DE GÉNÉRATION DES PERTURBATIONS. - Les perturbations électromagnétiques apparaissant durant la commutation résultent de deux effets :

- les conducteurs soumis à $\mathrm{d} V / \mathrm{d} t$ et $\mathrm{d} / / \mathrm{d} t$ deviennent sources de courants, tensions et champs parasites;

- le fonctionnement (commutation) du convertisseur détermine les régimes dynamiques de $\mathrm{d} V / \mathrm{d} t$ et $\mathrm{d} / / \mathrm{d} t$, donc toutes les excitations des circuits parasites.

Nous allons successivement examiner ces deux points.

2.3.1 Effets généraux des gradients de tension et courant sur un conducteur. - Les figures suivantes représentent le dispositif expérimental qui permet d'étudier le comportement d'un conducteur (piste de circuit imprimé) lorsqu'il est soumis à $\mathrm{d} V / \mathrm{d} t$ (Fig. 6a) ou $\mathrm{d} I / \mathrm{d} t$ (Fig. 6b).

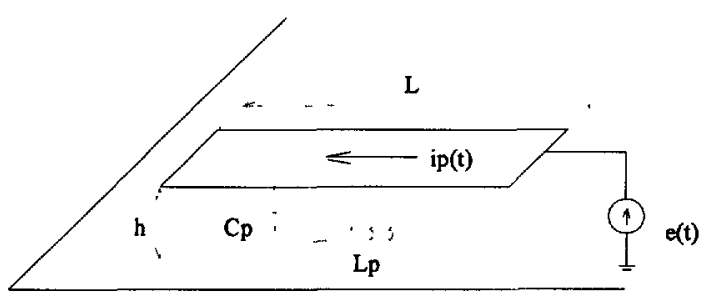

a)

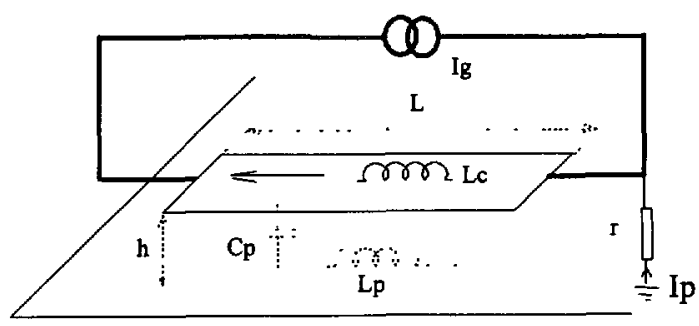

b)

Fig. 6. - a) Conducteur soumis à $\mathrm{d} V / \mathrm{d} t$. b) Conducteur soumis à $\mathrm{d} I / \mathrm{d} t$.

[a) $\mathrm{d} V / \mathrm{d} t$ applied on a conductor. b) $\mathrm{d} / / \mathrm{d} t$ applied on a conductor.] 
2.3.1.1 Effet d'un gradient de tension. - Le dispositif s'apparente à une ligne à constantes réparties. Ses dimensions restent petites devant la longueur d'onde des fréquences les plus élevées rencontrées (quelques mètres), il peut être modélisé par le schéma équivalent à constantes localisées de la figure 7. $L_{\mathrm{P}}$ et $C_{\mathrm{P}}$ sont les inductances et capacités parasites du conducteur vis-à-vis du plan de masse (il est possible de les connaître à partir des grandeurs linéiques), $e(t)$ est le générateur de tension d'attaque caractérisé par son $\mathrm{d} V / \mathrm{d} t(=a)$ et sa tension maximale $E$.
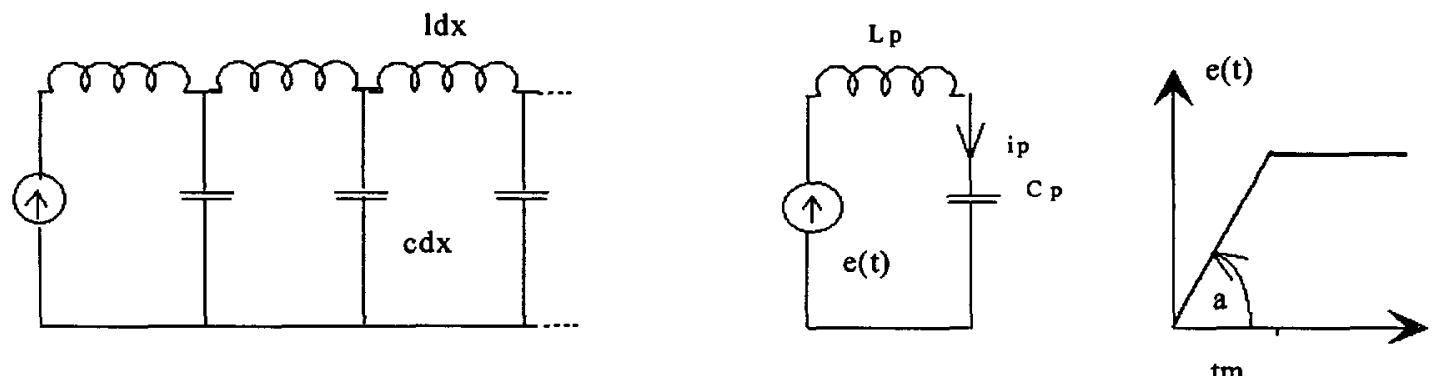

Fig. 7. - Modèle à composants localisés.

[Located components model.]

L'expression temporelle de la réponse $i_{\mathrm{p}}(t)$ s'écrit :

$$
i_{\mathrm{p}}(t)=a \cdot C_{\mathrm{p}} \cdot\{[U(t)-U(t-t m)]-[U(t) \cdot \cos (\omega t)-U(t-t m) \cdot \cos (\omega(t-t m))]\}
$$

avec :

$$
\omega=\frac{1}{\sqrt{L_{\mathrm{p}} \cdot C_{\mathrm{p}}}} .
$$

La réponse est constituée d'une impulsion de largeur $t m$ et d'un terme oscillatoire. Ce dernier est susceptible de s'annuler selon la valeur de $\mathrm{tm}$. La condition d'annulation s'écrit :

$$
\omega . t m=2 k \cdot \pi \text {. }
$$

On obtient une réponse unique d'amplitude $2 \cdot a \cdot C_{p}$ constituée d'une impulsion et d'une seule période de cosinus si $t m$ prend la valeur particulière:

$$
t m=\frac{2 \cdot \pi}{\omega}=T=2 \cdot \pi \cdot \sqrt{L_{\mathrm{p}} \cdot C_{\mathrm{p}}} .
$$

Ce mode constitue le régime de pollution minimale, comme le montrent les uıesures temporelles et spectrales du courant $i_{\mathrm{p}}$ (Fig. 8) pour plusieurs valeurs de $\mathrm{tm}$.

On met en évidence ici un phénomène de résonance propre au circuit de couplage de mode commun. Nous constatons que celui-ci est atténué si le gradient $\mathrm{d} V / \mathrm{d} t$ est adapté à ce circuit. Dans ce cas l'amplitude de $i_{\mathrm{p}}(t)$ ne dépend que de $E$ et de l'impédance caractéristique $Z_{c}$ de la ligne:

$$
i_{\mathrm{p}}=2 \cdot a \cdot C_{\mathrm{p}}=2 \cdot C_{\mathrm{p}} \frac{E}{t m}=\frac{E}{\pi} \cdot \sqrt{\frac{C_{\mathrm{p}}}{L_{\mathrm{p}}}}
$$



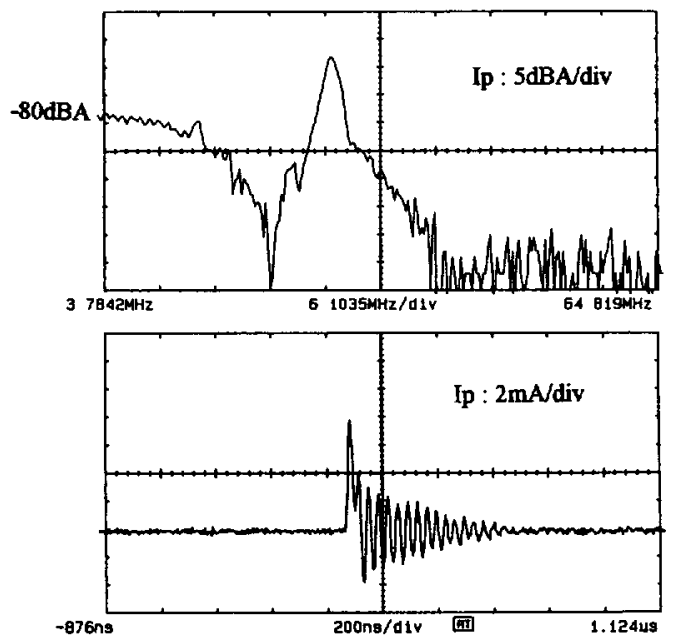

$\operatorname{tm}<\mathrm{T}$
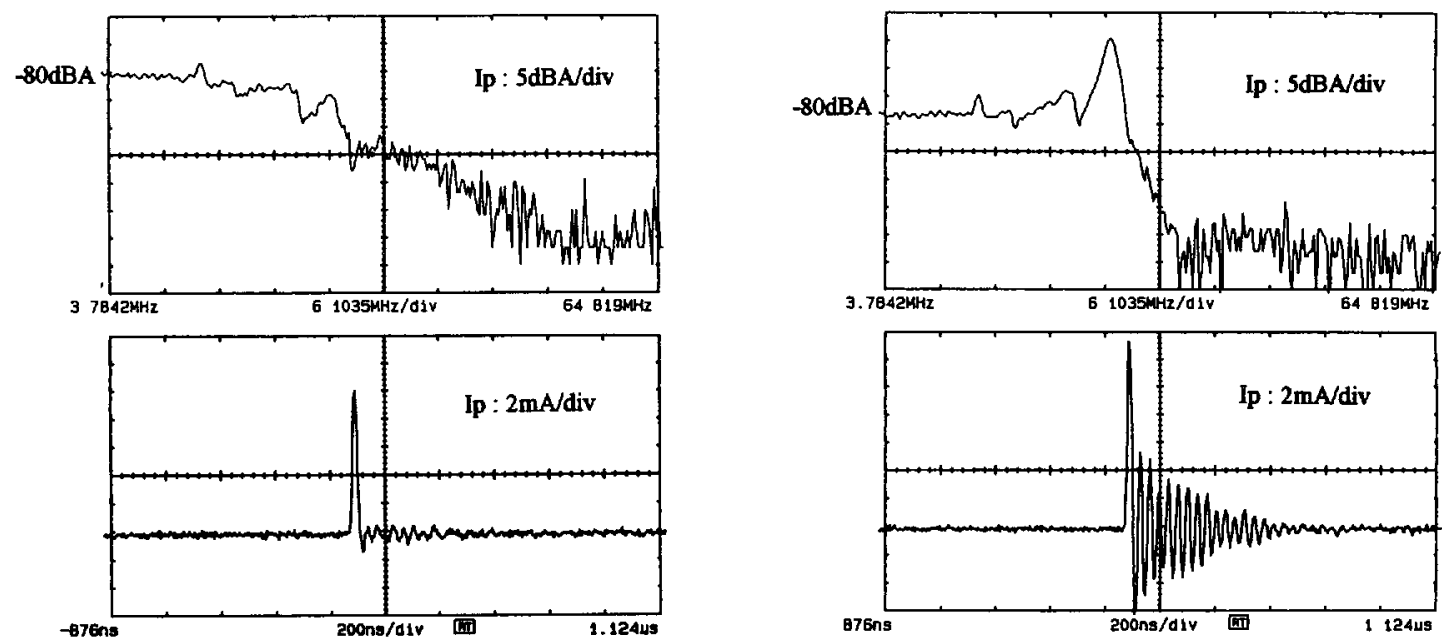

$\operatorname{tm}=\mathbf{T}$

$\operatorname{tm}>\mathrm{T}$

Fig. 8. - Résultats expérimentaux.

[Experimental results.]

avec :

$$
Z_{\mathrm{c}}=\sqrt{\frac{L_{\mathrm{p}}}{C_{\mathrm{p}}}}
$$

Il résulte de ces observations qu'il est nécessaire de réduire $C_{\mathrm{p}}$ et augmenter $L_{\mathrm{p}}$, c'est-à-dire éloigner le conducteur du plan de masse, en conservant la condition d'adaptation $t m=T$. 
2.3.1.2 Effet d'un gradient de courant. - Le conducteur, d'inductance parasite $L_{\mathrm{c}}$, situé audessus du plan de masse est cette fois soumis à un gradient $\mathrm{d} l / \mathrm{d} t$ de courant (schéma à constantes localisées de la Fig. 6b). Celui-ci va également produire un courant parasite $i_{\mathrm{p}}$ comme le montrent les courbes figure 9 .
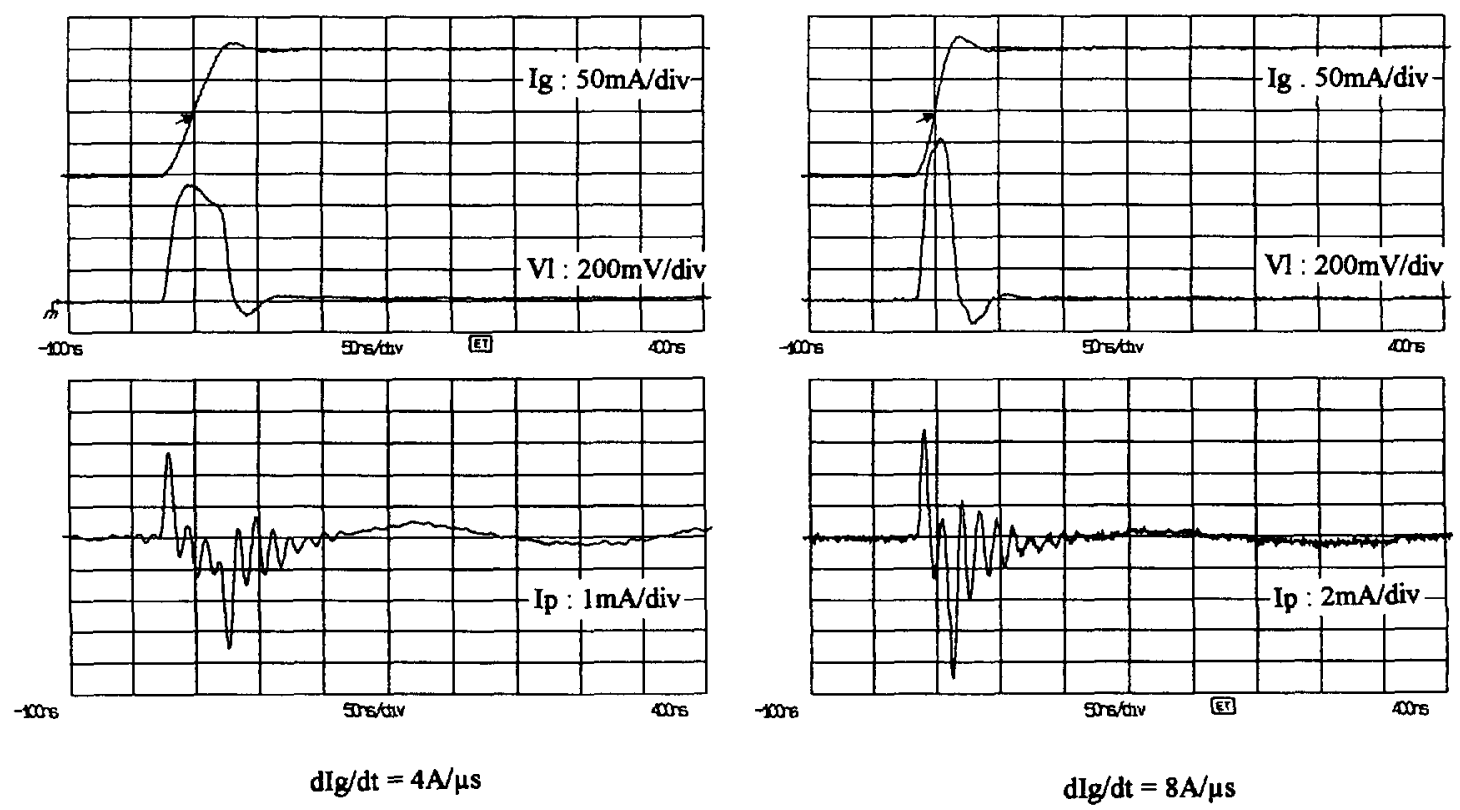

Fig. 9. - Réponses expérimentales.

[Experimental responses.]

Le gradient de courant crée une impulsion de tension aux bornes de l'inductance $L_{\mathrm{p}}$. Elle provoque ensuite la résonance du circuit $L_{\mathrm{p}} C_{\mathrm{p}}$ comme on l'a vu précédemment.

Le courant parasite $i_{\mathrm{p}}$ s'écrit :

$$
i_{\mathrm{p}}=\frac{p \cdot L_{\mathrm{c}} \cdot I_{\mathrm{g}}}{1+p \cdot r \cdot C_{\mathrm{p}}+p^{2} \cdot\left(L_{\mathrm{p}}+L_{\mathrm{c}}\right) \cdot C_{\mathrm{p}}}
$$

Si $I_{\mathrm{g}}$ est un trapèze de courant, le terme $p . L_{\mathrm{c}} \cdot I_{\mathrm{g}}$ constitue une impulsion de tension durant les variations de $I_{\mathrm{g}}$. $I_{\mathrm{p}}$ est la réponse du circuit à cette excitation, d'où les régimes oscillatoires complexes observés sur les courbes de la figure 9.

Les deux analyses qui précèdent peuvent s'appliquer aux conducteurs au sein d'un convertisseur. Les structures dans lesquelles les $\mathrm{d} V / \mathrm{d} t$ et $\mathrm{d} l / \mathrm{d} t$ sont réduits seront donc naturellement moins polluantes.

2.3.2 Mécanismes de création des perturbations lors des commutations [3, 4]. - Les régimes parasites qui se superposent au fonctionnement idéal des convertisseurs dépendent :

- des conditions initiales de courant et de tension dans les interrupteurs (transistor-diode) et des divers composants parasites les environnant aux instants de commutation, donc des modes de commutation ; 
- de l'environnement de l'interrupteur : la présence de circuits d'assistance en parallèle provoque l'apparition de multiples régimes parasites impliquant l'interrupteur. En effet, ces composants et leurs termes parasites (réactifs) sont sièges d'énergies résiduelles dont la résorbtion est à l'origine d'oscillations haute fréquence. La modélisation de ces phénomènes fait intervenir les composants parasites précédemment analysés. L'interrupteur est modélisé par un générateur de courant commandé associé avec une capacité $C_{\mathrm{k}}$ en parallèle et une inductance $\ell_{\mathrm{k}}$ en série.

Le tableau III réalise la synthèse des différentes étapes des commutations à l'amorçage et au blocage. On pourra constater que plus le nombre de composants en dérivation sur l'interrupteur est important et plus les régimes oscillatoires sont nombreux, compte tenu de l'augmentation des modes propres et des énergies résiduelles.

Les $\mathrm{d} / \mathrm{d} t$ qui apparaissent dans les structures résultent soit de la commande d'interrupteur, soit des recouvrements de diode. Leur effet est de provoquer l'excitation des différentes mailles peu amorties de la structure. On peut très sensiblement réduire le niveau de perturbation en ralentissant la commutation au détriment d'un accroissement des pertes par commutation.

La multiplicité des régimes transitoires devient très complexe et le calcul analytique des formes d'ondes est impossible. Il est donc nécessaire de recourir à la simulation numérique avec des logiciels de type circuit : SPICE ou SICOS [8].

Les courbes des figures 10 présentent les résultats de simulation comparés aux relevés expérimentaux. Le logiciel utilisé est SPICE, on peut constater la validité des modèles utilisés. Ces courbes concernent la phase de blocage du hacheur à commutation forcée en utilisant le modèle de la figure 5 , qui intègre les composants parasites précédemment décrits. On pourra remarquer que les différentes étapes sont correctement représentées ainsi que les pseudopulsations et les amplitudes des différents régimes parasites, confirmant l'approche analytique du tableau III.

\subsection{Mécanismes de Couplage.}

2.4.1 Couplages en mode conduit. - Les courbes de la figure 11 comparent les résultats expérimentaux et simulés du courant $i_{\mathrm{p}}$ de mode commun pour le hacheur à commutation forcée de la figure 5 . Ces résultats montrent la possibilité de prédéterminer par simulation le comportement CEM d'un convertisseur en utilisant une modélisation de type «circuit » à composants localisés. Nous avons pu confirmer l'étude analytique et mettre en évidence les principaux couplages parasites capacitifs responsables des perturbations conduites.

2.4.2 Couplages en mode rayonné. - Chaque maille d'un convertisseur, parcourue par un courant, va émettre au même titre qu'une antenne boucle un champ magnétique fonction de l'amplitude du courant, de la géométrie de la boucle et de son environnement immédiat. L'origine, la forme et l'évolution des courants d'excitation ont été établies au paragraphe précédent, nous allons ici développer plus spécifiquement le calcul du champ magnétique émis en tout point de l'espace en fonction de la géométrie de la boucle. L'examen des circuits électriques des convertisseurs statiques quelles que soient leur puissance et la technologie employée (circuit imprimé, etc.) montre que les formes occasionnées par les liaisons électriques décrivent des figures géométriques simples : des quadrilatères, aussi nous avons opté pour une modélisation rectangulaire des sources rayonnantes (Fig. 12).

Le calcul du champ $H$ est falt à partir des hypothèses suivantes :

- les conducteurs constituant les sources rayonnantes sont filiformes et leur résistivité est nulle ;

- la forme des boucles est rectangulaire ; 
Tableau III. - Les différents régimes parasites liés à l'interrupteur et à son proche environnement.

[Different parasitic modes of the switch in its near environment.]

\begin{tabular}{|c|c|c|c|c|}
\hline $\begin{array}{c}\text { (Interrupteur } \\
\text { et } \\
\text { son proche } \\
\text { environnement) }\end{array}$ & $\begin{array}{c}\text { BLOCAGE } \\
\text { Phase de } \\
\text { commutation }\end{array}$ & $\begin{array}{l}\text { Composants } \\
\text { impliqués }\end{array}$ & $\begin{array}{l}\text { AMORCAGE } \\
\text { Phase de } \\
\text { commutation }\end{array}$ & $\begin{array}{l}\text { Composants } \\
\text { impliqués }\end{array}$ \\
\hline $\begin{array}{c}\text { Commutation } \\
\text { forcée }\end{array}$ & $\begin{array}{c}\mathrm{vk} / \\
\text { Ecrêtage } \\
(\omega 1, \omega 2, \omega 3) \\
\\
\text { Réinit. } \\
(\omega 4) \\
\end{array}$ & $\begin{array}{c}\text { Ck, ly, lk } \\
C k, 1 k \\
\text { le, ly } \\
\text { Ck, le, lk } \\
\text { Ck, Ly, lc }\end{array}$ & $\begin{array}{c}\text { Recouvrement } \\
\text { de la Diode de } \\
\text { Roue libre }\end{array}$ & $\mathrm{Ck}, \mathrm{dIg} / \mathrm{dt}$ \\
\hline 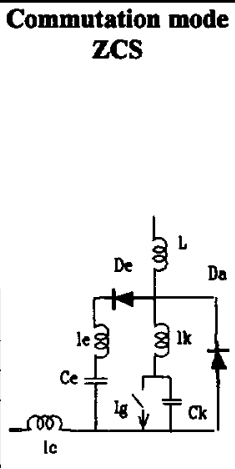 & $\begin{array}{c}\text { Ecrêtage } \\
(\omega 1, \omega 2) \\
\text { Réinit. } \\
(\omega 4)\end{array}$ & $\begin{array}{c}\text { Ck, L } \\
\text { Ck, le, lk } \\
\text { Ck, L, lc }\end{array}$ & Vk & $\mathrm{Ck}$, dIg/dt \\
\hline $\begin{array}{c}\text { Commutation mode } \\
\text { ZVS }\end{array}$ & ${ }_{(\omega 0)}{ }^{\mathrm{k}} 7$ & $\begin{array}{l}C k, l \gamma, \mathbf{l k} \\
\mathrm{C}, \mathrm{l} \gamma, \mathrm{lc}\end{array}$ & Vk & $\begin{array}{c}\mathrm{C} \gamma, \mathrm{l \gamma} \\
\mathrm{Ck}, \mathrm{lk}, \mathrm{l} \gamma\end{array}$ \\
\hline
\end{tabular}

Les différentes étapes transitoires qui constituent une commutation complète sont décrites ci-dessous :

$-v_{\mathrm{k}} /$ : remontée du potentiel d'interrupteur $\omega_{0}=\frac{1}{C_{\mathrm{k}}\left(\ell_{\mathrm{k}}+\ell_{\gamma}\right)}$

- $v_{\mathrm{h}}$ : descente du potentiel d'interrupteur.

- Ecrêtage : phase d'action de l'écrêteur. Durant cette phase trois régimes oscillatoires apparaissent :

$$
\omega_{1}=\frac{1}{C_{\mathrm{k}}\left(\ell_{\mathrm{k}}+\ell_{\mathrm{c}} / / \ell_{\gamma}\right)}, \quad \omega_{2}=\frac{1}{C_{\mathrm{k}}\left(\ell_{\mathrm{k}}+\ell_{\mathrm{e}}\right)}, \quad \omega_{3}=\frac{1}{C_{\mathrm{t}}\left(\ell_{\mathrm{e}}+\ell_{\gamma}\right)}
$$

$\left(C_{1}\right.$ est la capacité de transition de la diode $D_{y}$ )

- Réinit. : rétablissement de la tension de blocage de l'interrupteur : $\omega_{4}=1 / C_{\mathrm{k}}\left(L_{y}+\ell_{\mathrm{c}}\right)$. 


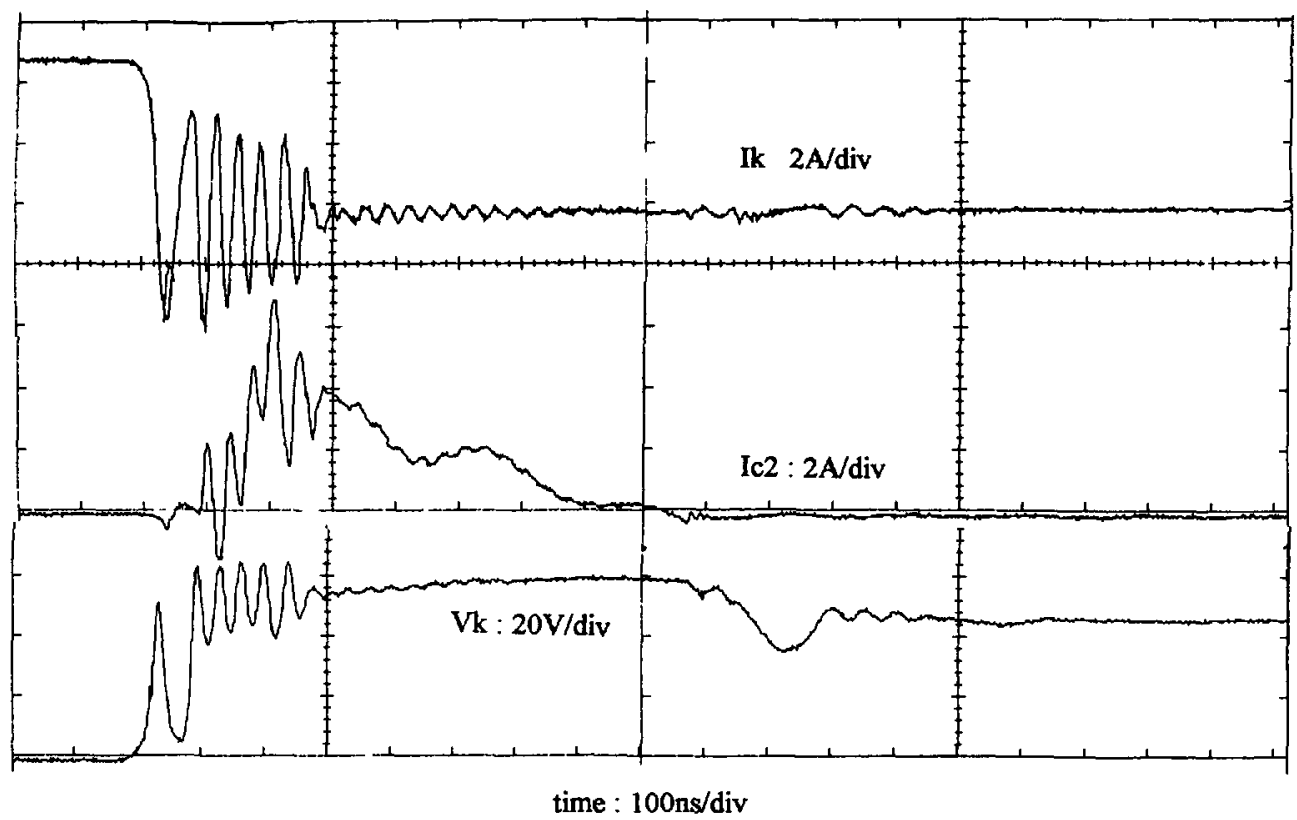

Experimental results

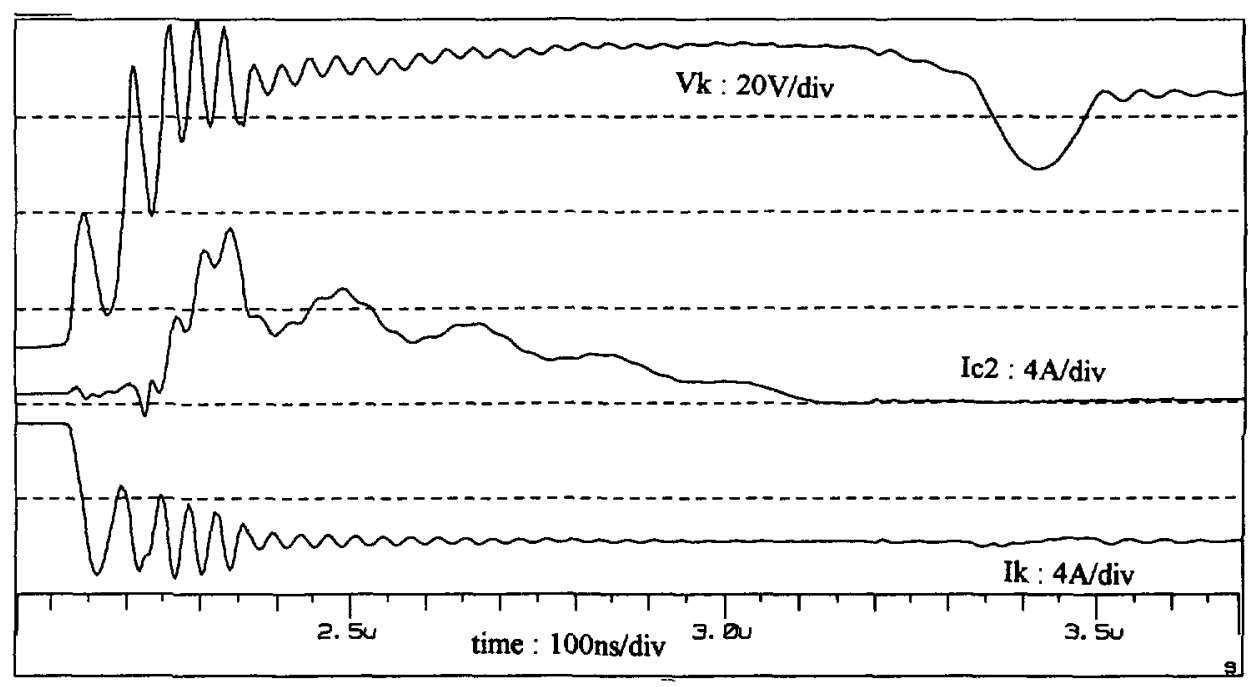

Simulation

Fig. 10. - Comparaison des résultats expérimentaux et simulés lors du blocage.

[Simulation results compared to experimental ones at switch-off.] 

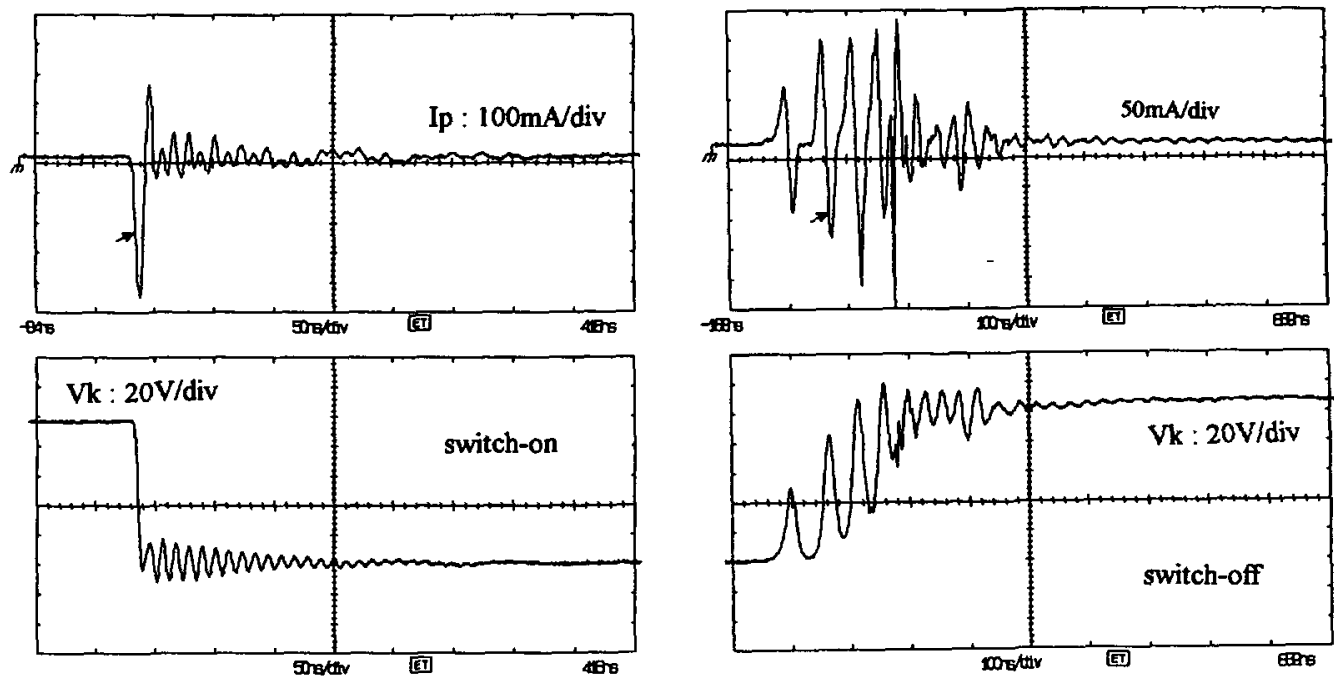

Experimental results
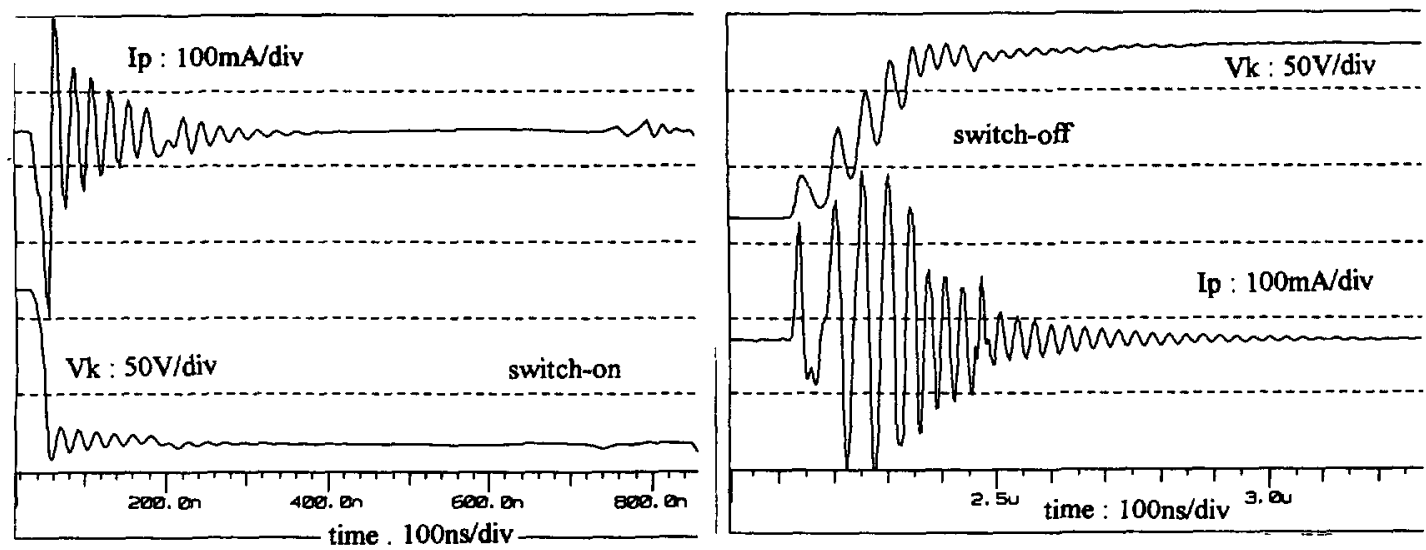

Simulation

Fig. 11. - Comparaison du courant de mode commun expérimental et simulé.

[Parasitic common mode current simulation results compared to experimental ones.]

- le rayon du cercle circonscrit à la maille est très inférieur à la longueur d'onde minimale du signal électrique d'excitation, ce qui implique une distribution de courant constante dans la boucle ;

- le champ $H$ se propage dans l'espace libre et le point d'observation est proche des sources ;

- le champ $H$ statique produit par un courant constant dans le temps, est exclu de cette étude, en effet il ne crée pas de tension induite dans le circuit victime. 


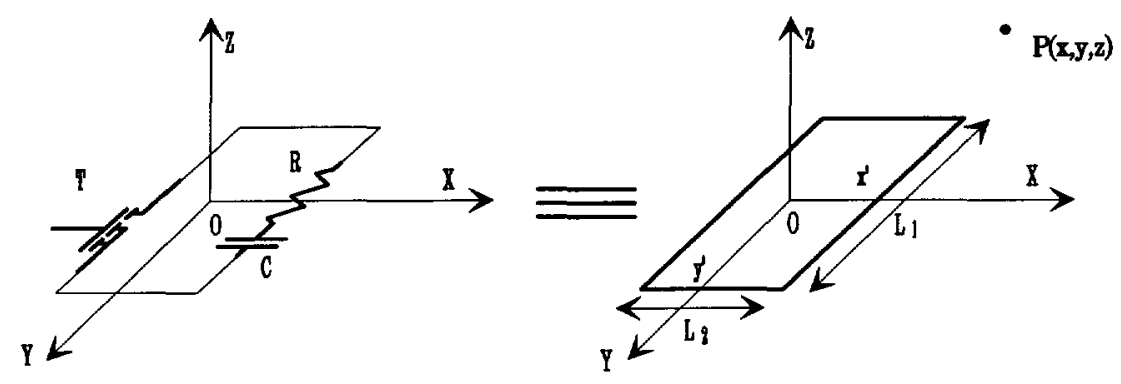

Fig. 12. - Modélısation d'une boucle rayonnante.

[Modelization of a radiating loop.]

A partir des équations de Maxwell appliquées à un milieu isotrope et homogène de permittivité $\varepsilon_{0}$ et de perméabilité $\mu_{0}$, le potentiel vecteur $A$ en tout point de cet espace en fonction du courant d'excitation est donné par l'expression :

$$
\mathbf{A}=\frac{\mu_{0}}{4 \pi} \int_{a}^{b} \frac{I \mathrm{~d} \ell^{\prime}}{\left[r-r^{\prime}\right]}
$$

Les coordonnées $r$ correspondent au point d'observation, tandis que les coordonnées $r^{\prime}$ dénotent la position des courants (Fig. 13). Le potentiel vecteur magnétique en un point $P(x, y, z)$ s'exprime de la façon suivante :

$$
\begin{aligned}
& A_{a \mathrm{a}}=\frac{\mu_{0} I}{4 \pi}\left[\int_{\frac{-L_{1}}{2}}^{\frac{L_{1}}{2}} \frac{\mathrm{A}=\mathbf{e}_{\imath} A_{a x}+\mathbf{e}_{y} A_{a y}}{\sqrt{\left(x-x^{\prime}\right)^{2}+\left(y+\frac{L_{2}}{2}\right)^{2}+z^{2}}}+\int_{\frac{L_{1}}{2}}^{\frac{-L_{1}}{2}} \frac{\mathrm{d} x^{\prime}}{\sqrt{\left(x-x^{\prime}\right)^{2}+\left(y-\frac{L_{2}}{2}\right)^{2}+z^{2}}}\right] \\
& A_{a v}=\frac{\mu_{0} I}{4 \pi}\left[\int_{\frac{L_{2}}{2}}^{\frac{-L_{2}}{2}} \frac{\mathrm{d} x^{\prime}}{\sqrt{\left(x-\frac{L_{1}}{2}\right)^{2}+\left(y-y^{\prime}\right)^{2}+z^{2}}}+\int_{\frac{-L_{2}}{2}}^{\frac{L_{2}}{2}} \frac{\mathrm{d} x^{\prime}}{\sqrt{\left(x+\frac{L_{1}}{2}\right)^{2}+\left(y-y^{\prime}\right)^{2}+z^{2}}}\right] .
\end{aligned}
$$

La mesure du champ magnétique $H$ étant réalisée à l'aide d'antennes boucles de différents diamètres, il faut donc trouver la fonction de couplage entre la source rectangulaire rayonnante et l'antenne de mesure. Pour ce faire, il suffit de calculer le champ magnétique moyen capté par l'antenne. Ce dernier s'exprime en fonction de la mutuelle inductance $M_{12}$ caractérisant ce couplage inductif entre les deux éléments. En appliquant les mêmes hypothèses que précédemment, il vient :

$$
H_{\text {moy }}=\frac{M_{12} i_{1}}{\mu_{0} S}
$$

avec $i_{1}$ courant circulant dans la source.

Le flux magnétique traversant l'antenne exprimé en fonction du potentiel vecteur magnétique engendré par la source autorise alors le calcul de cette mutuelle inductance

$$
M_{12}=\frac{1}{i_{1}} \int_{s} B \mathrm{~d} s=\frac{1}{i_{1}} \oint_{a} A \mathrm{~d} \ell .
$$




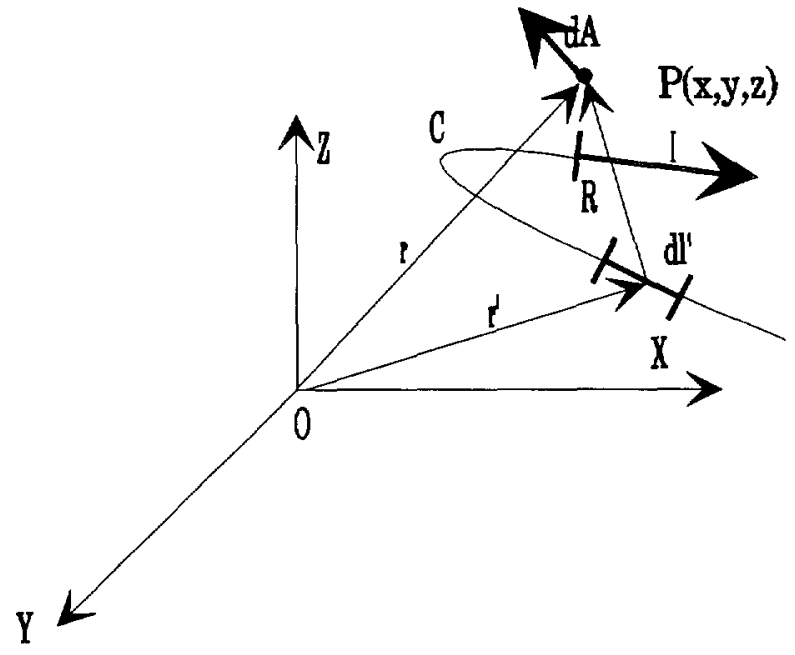

Fig. 13. - Potentiel vecteur d'un conducteur filiforme.

[Potentiel vector of a filiform current.]

La valeur de cette grandeur dépend de la position relative des deux mailles, la mesure dans deux directions orthogonales détermine la direction du champ magnétique, il suffit donc de connaître le champ quand les axes des deux mailles sont parallèles et perpendiculaires (Fig. 14).

En superposant l'axe de la boucle rectangulaire avec l'axe $\mathrm{O} z$ et celle-ci dans le plan $\mathrm{O} x y$, le potentiel vecteur dans le repère $O, x, y, z$ n'a pas de composante suivant $z$, en coordonnées cylindriques la mutuelle s'écrit :

- axes parallèles $M_{12}=\frac{1}{i_{1}} \int_{-\pi}^{\pi}\left(A_{a v} \cos \phi-A_{a r} \sin \phi\right) a \mathrm{~d} \phi$

- axes perpendiculaires $M_{12}=\frac{1}{i_{1}} \int_{-\pi}^{\pi} A_{a r} \sin \phi a \mathrm{~d} \phi$.

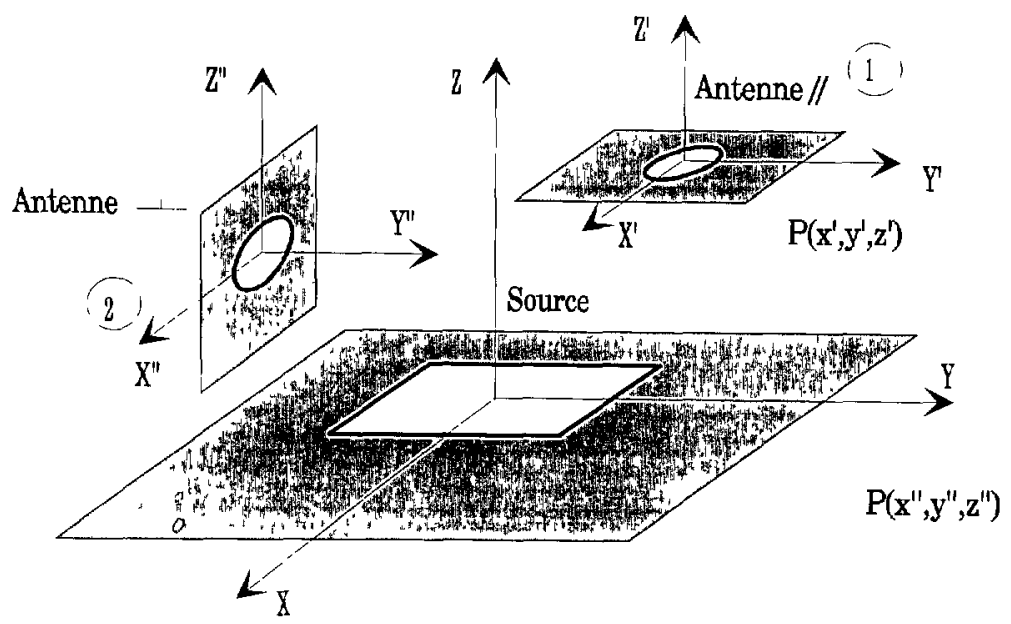

Fig. 14. - Couplages entre une source et une antenne: (1) parallèle, (2) perpendıculaire.

[Coupling between an antenna and a source, (1) parallel, (2) perpendicular.] 
La valeur du champ magnétique moyen est calculée à partir des expressions ci-dessus en résolvant des doubles intégrales numériquement par la méthode de Gauss.

2.4.3 Couplages en mode champ électrique. - Certaines parties du circuit électrique d'un convertisseur sont soumises à des variations rapides de la tension, le couplage capacitif avec la masse entraîne donc des courants de mode commun. Si les capacités de couplage sont de faibles valeurs et si l'impédance équivalente du circuit avec l'espace environnant est adapté il existe alors une possibilité de rayonnement en champ électrique $E$. Les branches, composant le circuit électrique, qui peuvent rayonner, sont modélisées par des monopoles excités par la variation temporelle de la tension à ses bornes lors des transitoires de commutation (Fig. 15).
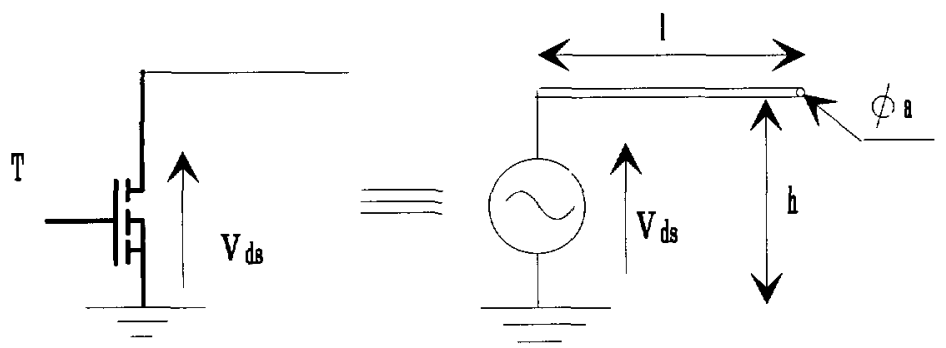

Fig. 15. - Caractérisation d'une source en champ $E$.

[Characterization of an $E$ field source.]

Ils sont caractérisés par leur longueur $\ell$, sa hauteur par rapport au plan de masse $h$ et son diamètre $a$. Afin de simplifier le calcul et les expressions du champ électrique, le conducteur antenne (ou monopole) est pris parallèle au plan de masse.

Le champ électrique rayonné, dans une région proche du conducteur, est fonction, outre des paramètres géométriques définis précédemment, de la distribution totale de la charge du conducteur. Elle se décompose en deux types de charge (Fig. 16) caractérisant le potentiel appliqué à une des extrémités du conducteur :

- une distribution linéique de charge $\rho_{1}$ constante et uniformément répartie le long du conducteur ;
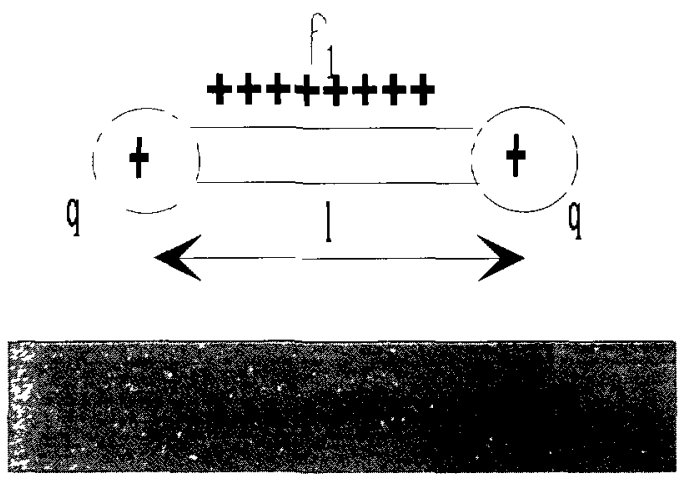

Fig. 16. - Distribution des charges sur un conducteur.

[Charge distribution along a conductor.] 
- une distribution de charge ponctuelle $q$ située aux deux extrémités du conducteur et traduisant la non-linéarité des bords.

La charge totale du conducteur et la valeur relative de la charge répartie par rapport à celle de la charge isolée sont données par les relations suivantes:

$$
Q=\ell \cdot \rho_{1}+2|q| \quad K(\ell)=\frac{\rho_{1} \ell}{q}
$$

On en déduit alors la densité linéique de charge et la valeur de la charge isolée :

$$
\rho_{1}=\left(\frac{K(\ell)}{K(\ell)+2}\right) \frac{Q}{\ell} \quad q=\left(\frac{\ell}{K(\ell)+2}\right) Q .
$$

L'évaluation de ce rapport $K$ en fonction de la longueur du conducteur $\ell$ a été faite à l'aide de deux logiciels de calcul électromagnétique à 3 dimensions, DIEL développé à EDF [9] et PHI3D développé au CEGELY [10].

La capacité du conducteur, placé en parallèle au plan de masse a été obtenue en faisant les hypothèses suivantes :

- le conducteur se situe dans l'espace libre $\left(\varepsilon=\varepsilon_{0}\right)$;

- le diamètre du conducteur $a$ est très faible devant sa longueur $\ell$, la charge électrique est concentrée dans l'axe du conducteur;

- le diamètre du conducteur $a$ est petit devant la distance séparant le conducteur de la masse $h$;

- le conducteur a une impédance nulle.

En supposant que la tension d'alimentation $V$ est égale au potentiel électrique du point $\mathrm{P}_{0}$ situé au centre du conducteur et sur sa surface, elle peut alors être calculée à partir de la charge électrique totale du conducteur $Q$

$$
C=\frac{Q}{V_{\mathrm{P}_{0}}}
$$

Le calcul de la tension s'exprime en fonction de la densité de charge électrique et des charges isolées, l'effet de masse est pris en compte par la théorie des images en plaçant un conducteur identique et de charge électrique égale et opposée

$$
\begin{array}{r}
V_{\mathrm{P}_{0}}=\frac{\rho_{1}}{2 \pi \varepsilon_{0}} \operatorname{Ln}\left[\frac{1}{a} \frac{2 h-a}{\frac{1}{2}+\sqrt{\left(\frac{1}{2}\right)^{2}+(2 h-a)^{2}}}\right]+\frac{q}{2 \pi \varepsilon_{0}} \frac{1}{\sqrt{\left(\frac{1}{2}\right)^{2}+a^{2}}}- \\
-\frac{q}{2 \pi \varepsilon_{0}} \frac{1}{\sqrt{\left(\frac{1}{2}\right)^{2}+(2 h-a)^{2}}}
\end{array}
$$

d'où la capacité du conducteur en fonction du coefficient $K(\ell)$ et en prenant en compte les hypothèses 2 et 3 :

$$
C=\frac{2 \pi \varepsilon_{0} \ell}{\operatorname{Ln} \frac{1}{a}+\operatorname{Ln} \frac{2 h}{\frac{1}{2}+\sqrt{\left(\frac{1}{2}\right)^{2}+4 h^{2}}}+\frac{2}{K(\ell)}} \frac{K(\ell)+2}{K(\ell)}
$$


La quantification du champ électrique $E$ est élaboré à partir de l'équation de Coulomb :

$$
\mathbf{E}=\frac{1}{4 \pi \varepsilon_{0}}\left[\int_{c^{\prime}} \frac{\mathbf{r}-\mathbf{r}^{\prime}}{\left|\mathbf{r}-\mathbf{r}^{\prime}\right|^{3}} \rho_{1} \mathrm{~d} \boldsymbol{\ell}^{\prime}+\sum_{k} \frac{\mathbf{r}-\mathbf{r}_{k}}{\left|\mathbf{r}-\mathbf{r}_{k}\right|^{3}} q_{k}\right] .
$$

En remplaçant la densité de charge et les charges ponctuelles par leur valeur en fonction de la tension $V$ et de la capacité du conducteur, l'expression analytique du champ $E$ dans le plan $\mathrm{O}, x, z$ contenant le conducteur et son image par rapport au plan de masse est égale à :

$$
\begin{aligned}
& E_{1}=\frac{C V}{4 \pi \varepsilon_{0}} \frac{x}{K(\ell)+2}\left[\frac{K(\ell)}{x}\left[\frac{z+\frac{1}{2}}{\sqrt{x^{2}+\left(z+\frac{1}{2}\right)^{2}}}-\frac{z-\frac{1}{2}}{\sqrt{x^{2}+\left(z-\frac{1}{2}\right)^{2}}}\right]+\right. \\
& \left.+\left[\frac{1}{\left[\sqrt{x^{2}+\left(z+\frac{1}{2}\right)^{2}}\right]^{3}}+\frac{1}{\left[\sqrt{x^{2}+\left(z-\frac{1}{2}\right)^{2}}\right]^{3}}\right]\right] \\
& E_{-}=\frac{C V}{4 \pi \varepsilon_{0}} \frac{1}{K(\ell)+2}\left[\frac{K(\ell)}{x}\left[\frac{1}{\sqrt{x^{2}+\left(z-\frac{1}{2}\right)^{2}}}-\frac{1}{\sqrt{x^{2}+\left(z+\frac{1}{2}\right)^{2}}}\right]+\right. \\
& \left.+\left[\frac{z+\frac{1}{2}}{\left[\sqrt{x^{2}+\left(z+\frac{1}{2}\right)^{2}}\right]^{3}}+\frac{z-\frac{1}{2}}{\left[\sqrt{x^{2}+\left(z-\frac{1}{2}\right)^{2}}\right]^{3}}\right]\right] .
\end{aligned}
$$

\subsubsection{Quelques résultats sur le rayonnement.}

\subsubsection{Localisation des sources.}

En champ magnétique. - La mesure montre que l'étage de commande produit un champ magnétique négligeable, par contre l'étage de puissance crée un champ magnétique notable au droit des boucles représentées à la figure 17 .

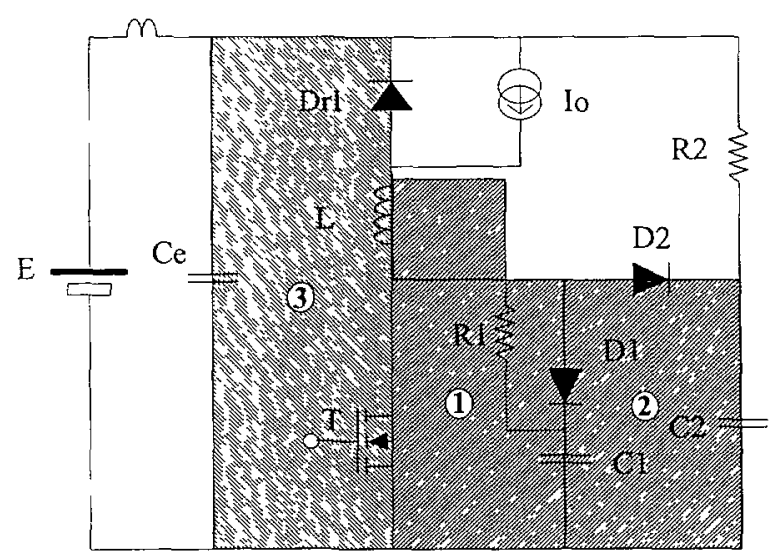

Fig. 17. - Différentes boucles rayonnantes.

[Different radiating loops.] 
La boucle 1 est constituée par le transistor MOS associé à son circuit d'aide à l'ouverture. La boucle 2 est également formée avec le transistor MOS et son circuit écrêteur. Enfin, la boucle 3 se compose toujours du même transistor, de la diode de roue libre, de l'inductance $L$ circuit d'aide à l'amorçage et du condensateur de découplage de la source. Le rayonnement n'est pas continu, il se produit pendant la commutation du transistor de puissance :

- à la fermeture, les mailles 1 et 2 émettent faiblement, ceci étant dû essentiellement à la vitesse de décharge de la capacité interne Cds du MOS,

- à l'ouverture, la décroissance du courant dans le MOS étant très rapide, il vient alors, exciter les éléments constituant les mailles précédentes et son évolution dans le temps se présente comme une sinusoïde HF décroissante (voir l'allure du courant $I_{k}$ Fig. 11).

Le champ qui en résulte, est très important. La figure 18 montre la comparaison entre le champ calculé à partir du courant dans le MOS, et celui mesuré au-dessus des boucles 1 et 2 dans un plan parallèle à $50 \mathrm{~mm}$ de distance.
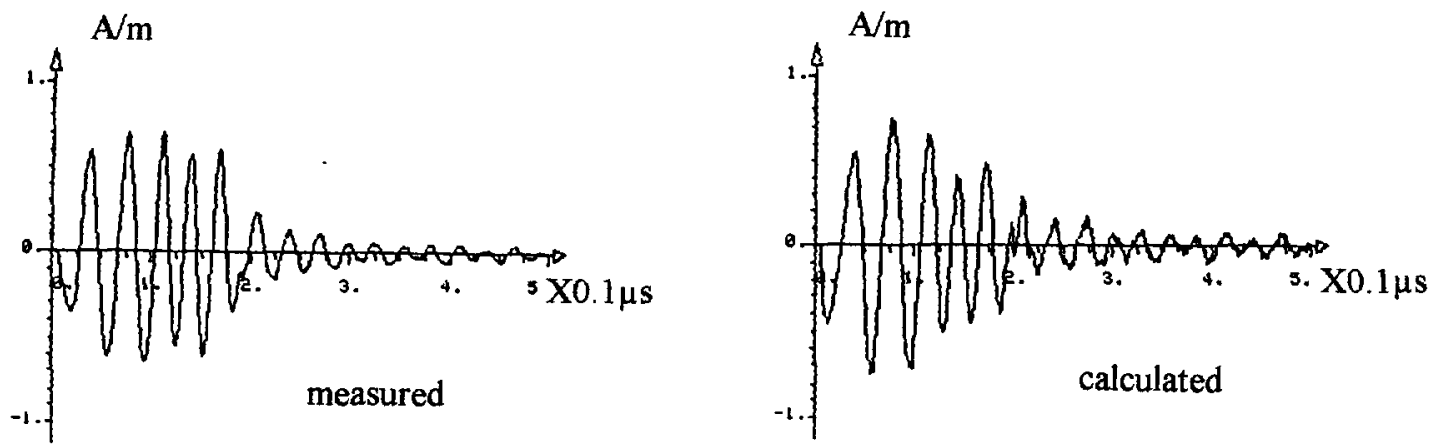

Fig. 18. - Champ $H(\mathrm{~A} / \mathrm{m})$ mesuré et calculé au blocage au-dessus des boucles $\mathrm{n} 1$ et $\mathrm{n} 2$.

[ $H$ field $(\mathrm{A} / \mathrm{m})$ measured and calculated at switch-off above loops $\mathrm{n} 1$ and $\mathrm{n} 2$ 2.]

- Indépendamment de la nature de la commutation, la boucle 3 produit un champ très important, mais à fréquence plus basse à la fermeture, cela étant dû au courant inverse de la diode de roue libre qui stimule la maille présentant une fréquence propre plus faible comparativement aux autres (Fig. 19).

La bonne corrélation entre les grandeurs calculées et mesurées permet de valider cette méthodologie.
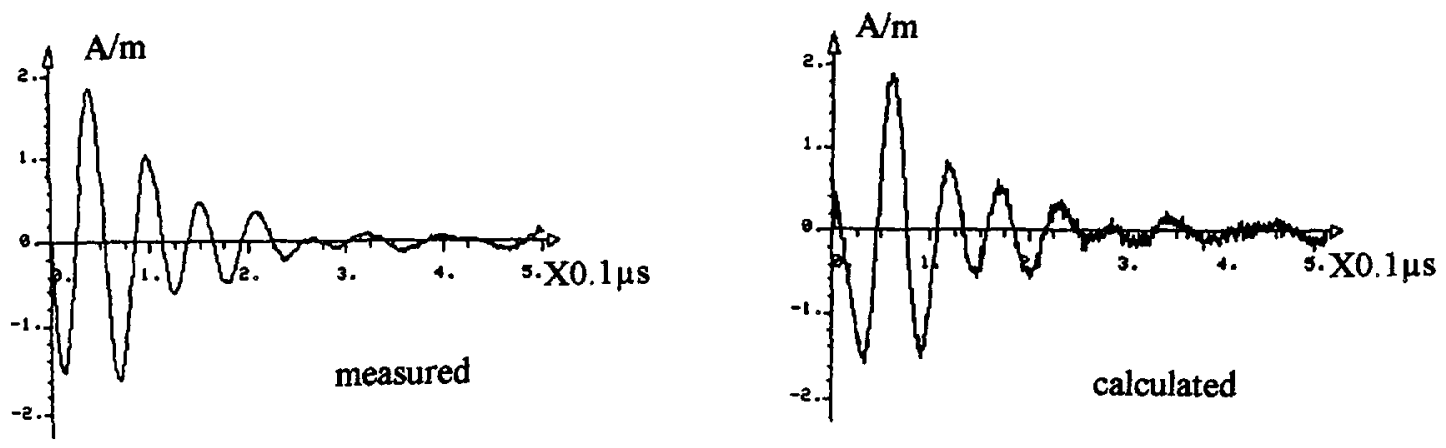

Fig. 19. - Champ $H(\mathrm{~A} / \mathrm{m})$ mesuré et calculé au-dessus de la boucle n 3 .

[H field $(\mathrm{A} / \mathrm{m})$ measured and calculated above man loop $\mathrm{n}$ 3.] 
En champ électrique. - Les sources en champ électrique sont aussi contiguës à la cellule de commutation. Tous les conducteurs reliés au drain du MOS (point chaud du circuit) font office d'antenne (Fig. 20), on nomme cette source l'arbre de rayonnement. Leur mécanisme de rayonnement est identique à celui du monopole excité par la variation de la tension $V \mathrm{~d} s$ aux bornes du transistor. Les autres conducteurs étant reliés en régime dynamique au potentiel de masse du circuit, produisent un champ électrique négligeable.

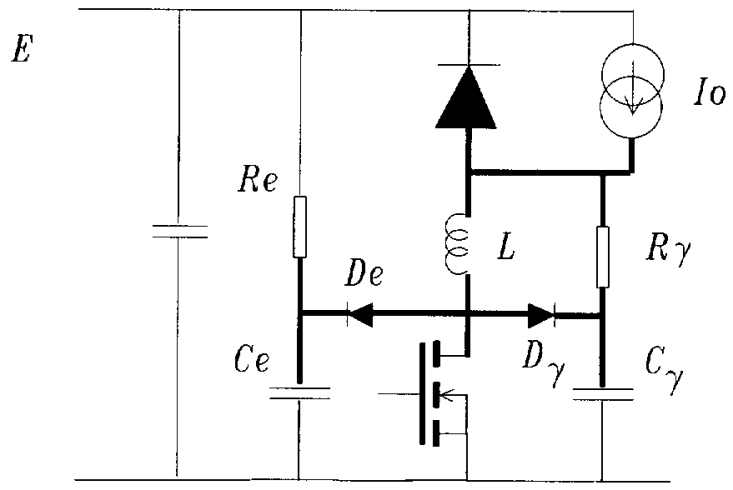

Fig. 20 - Arbre de rayonnement en champ $E$.

[E field radiating tree.]

Pour illustrer ce propos, la figure 21 montre une comparaison entre le champ $E$ mesuré audessus de la médiatrice du conducteur reliant le transistor de puissance à la diode $D e$ connectée à l'écrêteur et le champ calculé à partir de la tension aux bornes du transistor de puissance lors du blocage. Les résultats obtenus confirment correctement l'étude analytique.

\section{Evaluation quantitative des perturbations électromagnétiques.}

3.1 OUTILS D'ÉVALUATION DES PERTURBATIONS : APPROCHE ÉNERGÉTIQUE. - La représentation traditionnelle des mesures en CEM consiste à mesurer le spectre du signal parasite (courant ou champ). Celui-ci doit s'inscrire en-deçà d'un gabarit lorsque l'équipement est utilisé dans ses conditions nominales. Cette méthode présente les inconvénients suivants :

- difficultés d'interprétation selon les régimes de fonctionnement électrique (modification de la charge ou de la tension d'alimentation, modes de commutation);

- niveau de spectre lié à la valeur de la fréquence de découpage, donc ne représentant pas réellement son pouvoir perturbateur;

- impossibilité de séparation des phénomènes à l'amorçage et au blocage.

Nous proposons une méthode temporelle applicable aux signaux parasites de mode commun ou de champ électromagnétiques. Ces signaux sont nuls sauf durant les transitoires de commutation. Aussi est-il possible d'évaluer leur énergie (au sens du traitement du signal) à ces instants (Fig. 22)

$$
\begin{aligned}
& W_{\mathrm{p}}^{*}=\int_{t_{\mathrm{com}}} i_{\mathrm{p}}^{2}(t) \cdot \mathrm{d} t \text { en } \mathrm{A}^{2} \cdot \mathrm{s} \\
& W_{\mathrm{H}}^{*}=\int_{t_{\mathrm{com}}} H^{2}(t) \cdot \mathrm{d} t \text { en } \mathrm{A}^{2} \cdot \mathrm{s} / \mathrm{m} .
\end{aligned}
$$




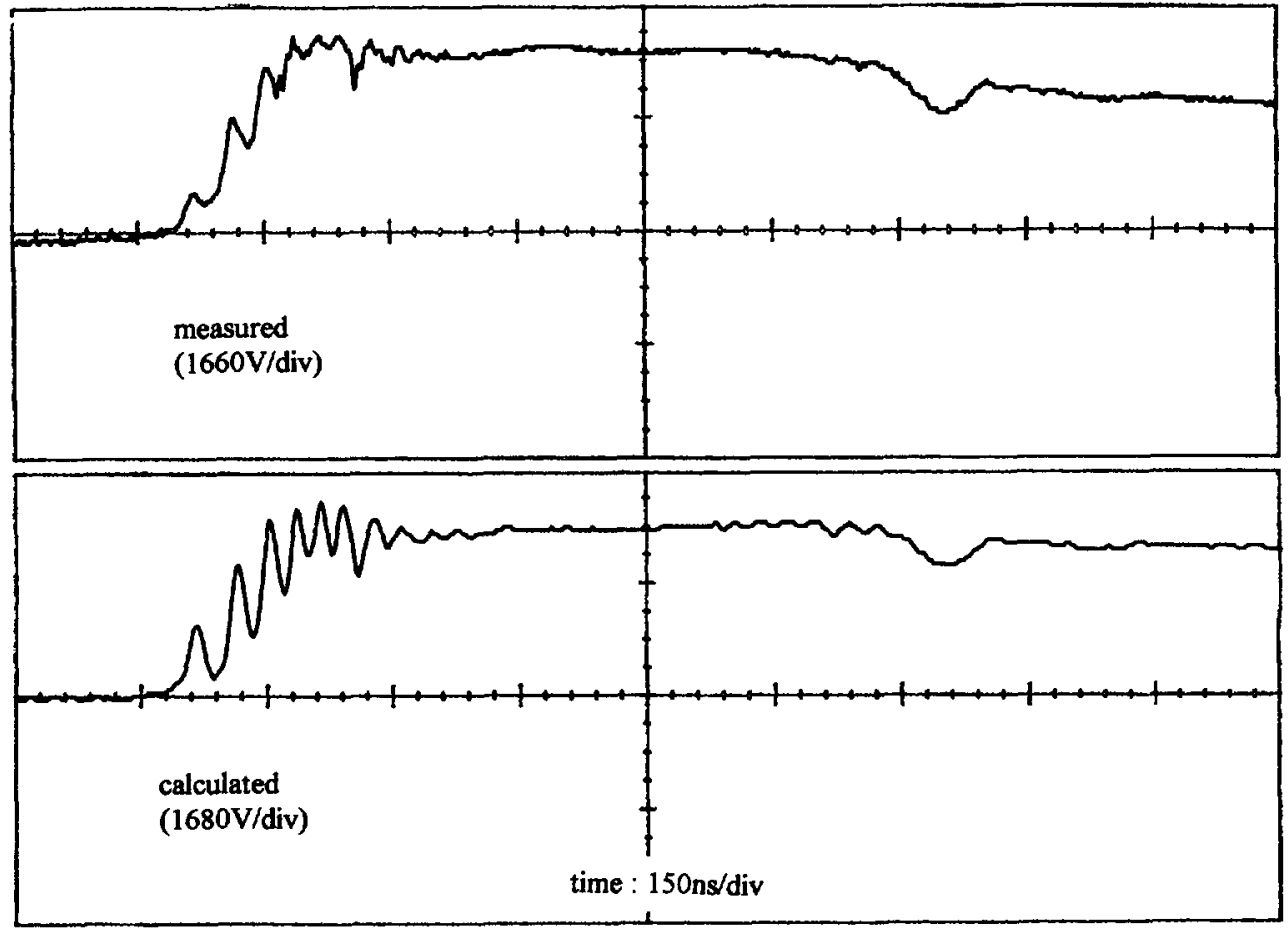

Fig. 21. - Champ $E(\mathrm{~V} / \mathrm{m})$ mesuré et calculé au-dessus du conducteur relié à l'interrupteur.

[E field $(\mathrm{V} / \mathrm{m})$ measured and calculated above the conductor linked to the switch.]

Cette méthode est réalisable par acquisition et traitement numérique du signal. On peut ainsi caractériser chaque commutation (amorçage : $W_{\text {on }}$, blocage : $W_{\text {off }}$ ), l'évolution en fonction des grandeurs électriques (tension d'alimentation, courant de charge, vitesse de commande de l'interrupteur) et établir des comparaisons quantitatives et qualitatives quant aux modes de commutation. L'énergie réelle $W_{\mathrm{p}}$ (ou $W_{\mathrm{H}}$ ) est obtenue à un facteur résistif (et géométrique) près.

La méthode présente toutefois l'inconvénient de fournir des résultats liés à la topologie du circuit de fermeture des courants de mode commun ou à la position de mesure des champs rayonnés. Dans nos essais, l'impédance de fermeture du courant de mode commun est déterminée par le RSIL et vaut $50 \Omega$. Cependant la même remarque peut s'appliquer aux méthodes traditionnelles de mesure (où l'environnement est défini par des normes). Les mesures présentées ont donc été réalisées systématiquement avec le même environnement.

\subsection{RÉSUltats OBTENUS.}

3.2.1 Application à une cellule de commutation simple. - Nous allons montrer que les énergies parasites $W_{\mathrm{p}}$ et $W_{\mathbf{H}}$ sont des fractions de l'énergie résiduelle des composants inductifs (ou capacitifs) présentes dans le convertisseur lors des phases de commutation.

La figure 23 présente un modèle de cellule de commutation simple dans laquelle on a fait apparaître les inductances et capacités parasites essentielles, ainsi que les impédances de mode commun. Le transistor est modélisé par un interrupteur binaire et une résistance $R d s o n$, on suppose son temps de commutation petit devant les constantes de temps du système. 


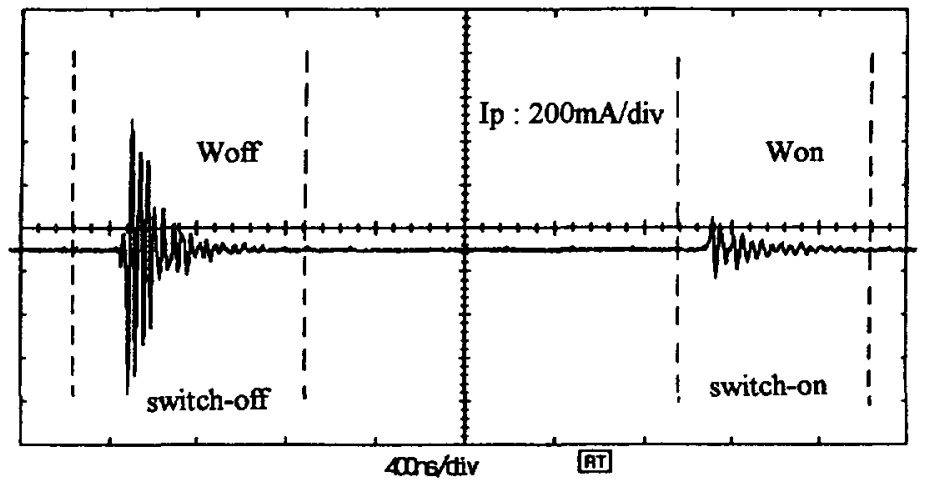

Fig. 22. - Définition de l'énergie du signal parasite.

[Definition of the energy of interference signal.]

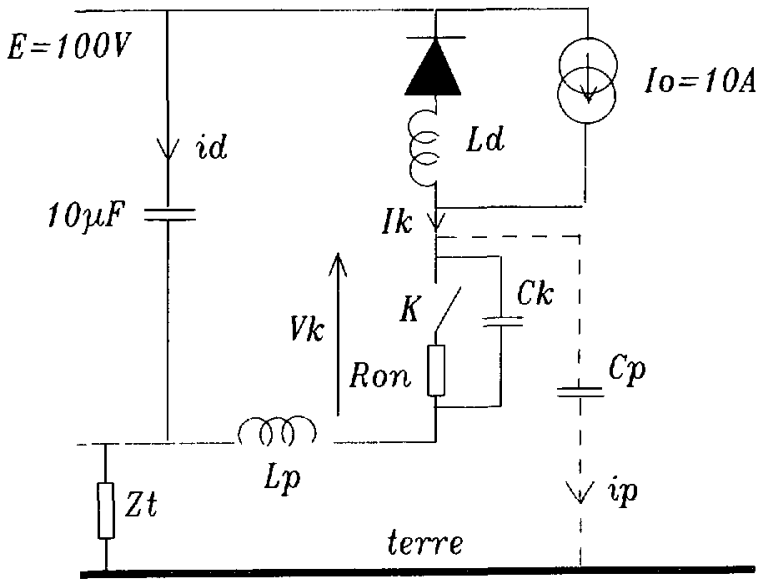

Fig. 23. - Modèle simplifié de la cellule de commutation.

[Simplıfied model for a switching cell.]

Le blocage rapide de l'interrupteur (et conséquemment l'amorçage de la diode) provoque l'apparition de Ia capacité parasite d'interrupteur $C_{\mathrm{k}}$. Il subsiste une énergie résiduelle dans $L_{\mathrm{t}}=L_{\mathrm{p}}+L_{\mathrm{d}}$ qui tend à s'annuler :

$$
W_{\mathrm{res}}=\frac{1}{2} \cdot L_{\mathrm{t}} \cdot I_{0}^{2}
$$

Celle-ci engendre un régime oscillatoire $\left(L_{\mathrm{t}} \cdot C_{\mathrm{k}}\right)$ de courant de mode différentiel $I_{\mathrm{d}}$ et amorti par la résistance faible de la diode et du câblage. On constate donc que le courant $I_{0}$ commuté par la cellule détermine principalement ces régimes parasites.

A l'amorçage c'est la présence de la capacité parasite de transition de la diode bloquée $C_{\mathrm{t}}$ qui génère le régime oscillatoire $\left(L_{\mathrm{t}} \cdot C_{\mathrm{t}}\right)$. L'énergie résiduelle à résorber vaut :

$$
W_{\mathrm{res}}=\frac{1}{2} \cdot L_{\mathrm{t}} \cdot I_{\mathrm{rm}}^{2} \text {. }
$$


Le régime oscillatoire présente la même forme que plus haut, il est amorti par $R d s o n$. Le régime parasite $I_{\mathrm{d}}$ est donc essentiellement déterminé par $\mathrm{Irm}$ dans cette phase. Nous avons pu vérifier que le courant $\mathrm{Ir} m$ est une fonction de la racine carrée de $I_{0}$.

$$
I r m=\sqrt{2 \cdot \frac{\mathrm{d} i_{\mathrm{k}}}{\mathrm{d} t} \cdot k \cdot I_{0}} .
$$

L'évolution de ce régime est alors dépendante $\mathrm{du}(\mathrm{d} i / \mathrm{d} t)$ de mise en conduction, donc de la commande de l'interrupteur et de la racine carrée du courant de charge.

La présence de composants parasites de couplage $\left(C_{p}, Z_{\mathrm{t}}\right)$ va permettre le transfert d'une partie du courant de mode différentiel $I_{\mathrm{d}}$ en mode commun :

- au blocage, une fraction du courant Imd est dérivée via $C_{\mathrm{p}}$ et $Z_{\mathrm{t}}$. On constate donc une évolution de $W_{\text {on }}$ proportionnelle à $I_{0}^{2}$;

- à lamorçage, $W_{\text {res }}$, donc $W_{\text {off }}$, dépend de $l \mathrm{rm}^{2}$. Nous avons constaté que le courant $I r m$ est sensiblement proportionnel à la racine carrée de $I_{0}$. Il s'ensuit donc que $W_{\mathrm{p}}$ évolue quasiproportionnellement à $I_{0}$. Lorsque $I_{0}=0$, c'est la variation rapide de $V_{\mathrm{h}}$ qui modifie la charge de $C_{p}$. L'énergie $W_{\text {off }}$ associée est constante (ordonnée à l'origine de la courbe) et vaut:

$$
W_{\mathrm{p}}=\frac{1}{2} \cdot C_{\mathrm{p}} E^{2}
$$

Les courbes de la figure 24 présentent l'évolution de l'énergie parasite $W_{\mathrm{p}}$ corrélativement au courant de charge $I_{0}$ dans la cellule de commutation. Elles confirment l'analyse faite.

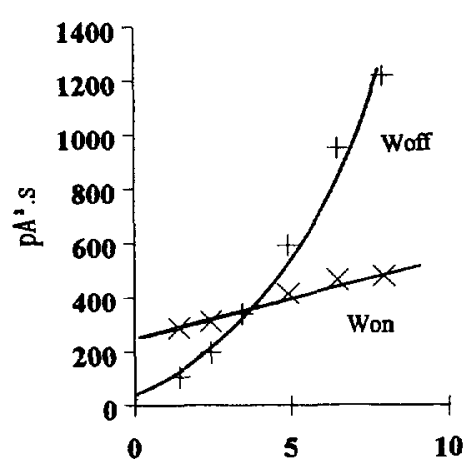

(A)

Fig. 24. - Evolution de $W_{\text {on }}$ et $W_{\text {nft }}$ en fonction du courant de charge $I_{0}$.

[Evolution of $W_{\text {on }}$ and $W_{\text {oft }}$ with load current.]

Il apparaît de cette analyse que le mode de commutation de la cellule, la présence de divers composants parasites, la vitesse de commande, déterminent son régime de pollution électromagnétique. D'autre part, l'influence de la fréquence de conmutation se traduit par une injection proportionnelle des courants parasites dans les circuits extérieurs.

3.2.2 Application aux différents modes de commutation. - A partir de l'analyse précédemment faite, on va pouvoir déterminer plus précisément les facteurs polluants liés aux différents modes de commutation. 
- La commutation forcée apparaît théoriquement polluante.

- La commutation à zéro de courant (ZCS) paraît intéressante, ayant lieu à courant nul dans l'interrupteur, il en est de même des énergies résiduelles tant à l'amorçage qu'au blocage.

- La commutation à zéro de tension (ZVS) apparaît polluante lors de la commande au blocage, mais on peut la ralentir sans trop augmenter les pertes.

Les courbes de la figure 25 montrent l'évolution de l'énergie au blocage et à l'amorçage pour les hacheurs à commutation forcée et quasi-résonnants (ZCS), en fonction de la tension d'alimentation $E$ à courant de charge $I_{0}$ constant, puis en fonction du courant de charge $I_{0}$ à tension d'alimentation $E$ constante. On constate des différences d'évolution notables selon les modes de commutation. On peut en tirer les interprétations suivantes :

- $W_{\text {on }}$ varie peu en fonction de $I_{0}$ en mode ZCS. En effet. 1'inductance de résonance limite fortement le $\mathrm{d} I_{\mathrm{k}} / \mathrm{d} t$ à la mise en conduction et le courant parasite de mode commun est essentiellement dû au $\mathrm{d} V_{\mathrm{k}} / \mathrm{d} t$. Cet effet se confirme lorsque l'on fait évoluer $E$ à

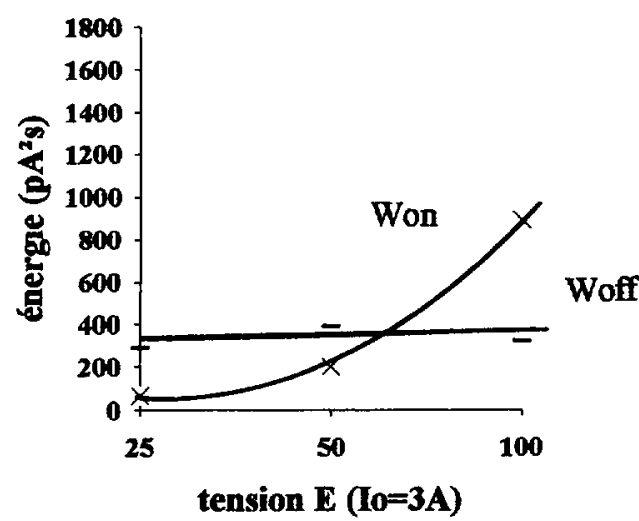

Hacheur à commutation forcée

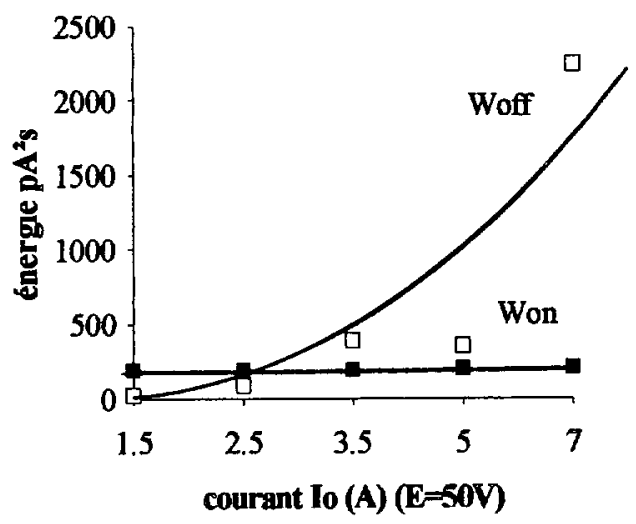

Hacheur à commutation forcée

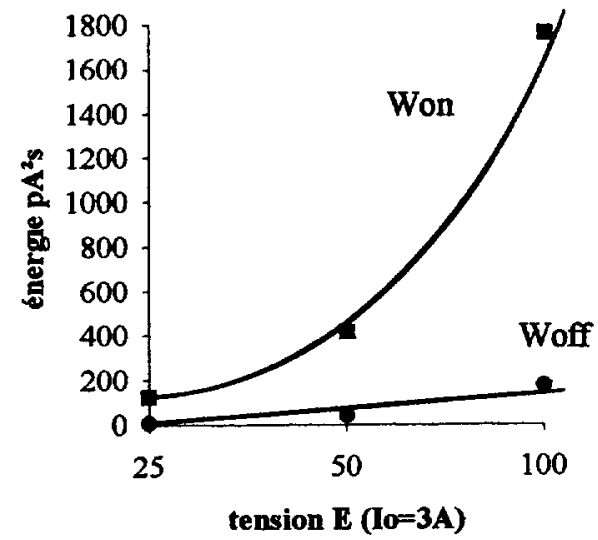

Hacheur quasi-résonnant

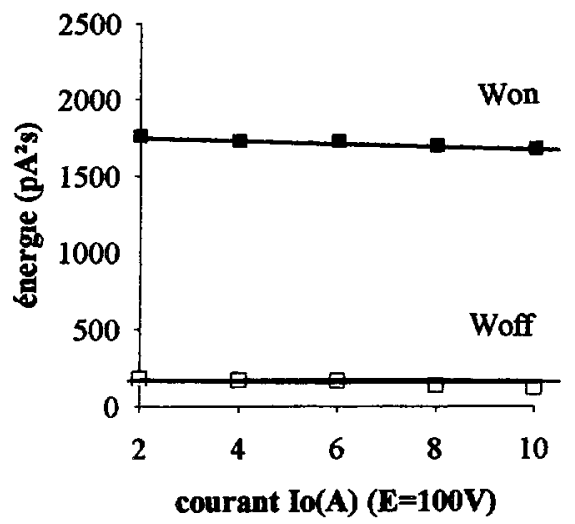

Hacheur quasi-résonnant

Fig. 25. - Evolution de l'énergie $W_{\text {on }}$ et $W_{\text {oft }}$ en fonction des grandeurs électriques.

[Evolution of energy $W_{\text {on }}$ and $W_{\text {olf }}$ with electrical constraints.] 
$I_{0}$ constant : l'énergie évolue sensiblement selon le carré de $E$. On retrouve ce comportement en commutation forcée où la phase d'amorçage assistée ressemble à celle du mode ZCS.

- $W_{\text {off }}$ est fortement variable en fonction de $I_{0}$ en commutation forcée, elle est par ailleurs relativement peu sensible à la tension $E, I_{0}$ étant fixé. On peut interpréter cette observation par le fait que dans ce mode, l'énergie résiduelle présente dans les inductances parasites des différentes branches dépend du niveau de courant lors du blocage. Durant cette phase, cette énergie est transférée aux différentes capacités parasites, selon des régimes oscillatoires complexes et multiples dépendant du nombre de branches soumises à $\mathrm{d} / / \mathrm{d} t$, d'où la sensibilité de la perturbation produite en courant commuté.

Inversement en mode $\mathrm{ZCS}$, l'annulation spontanée du courant dans la maille principale de commutation ainsi que le nombre plus faible de branches inductives soumises à $\mathrm{d} / / \mathrm{d} t$ rend cette commutation quasiment insensible à $I_{0}$ comme le montre la courbe $W_{\text {off }}=f\left(I_{0}\right)$. On constate même une légère diminution au point nominal, due à l'allégement de la contrainte de recouvrement de la diode antiparallèle à ce point.

3.2.3 Evaluation quantitative [3,4], - En plus de l'évaluation précitée, nous avons effectué des mesures spectrales sur les courants conduits et champs rayonnés par les différents prototypes, nous en avons dégagé les tendances suivantes :

A propos des modes de commutation. - Les mesures effectuées ont permis de constater que les structures à commutation douces présentent le niveau de perturbation le plus faible à leur point nominal. En effet dans chaque cas, ce point de fonctionnement correspond à la contrainte la plus réduite de la commutation non assistée :

- commutation au blocage à courant quasi-nul en mode ZVS ;

- commutation au blocage pour le mode ZCS sans recouvrement de diode.

Dans chaque cas les énergies résiduelles dans les composants parasites inductifs sont minimales, limitant les régimes oscillatoires.

11 subsiste toutefois un transitoire «dur» sur $V_{\mathrm{k}}$ à l'amorçage de l'interrupteur en mode ZCS, mais celui-ci peut être facilement contrôlé par la commande, sans pertes supplémentaires significatives [11], tout en réduisant considérablement la pollution de mode commun. La structure quasi-résonnante présente un comportement favorable concernant la minimisation des courants de mode différentiel.

La structure résonnante (ZVS) est favorable à la minimisation des courants de mode commun par le contrôle symétrique des gradients de potentiel $V_{\mathrm{k}}$ et à condition de ralentir les commandes d'interrupteur.

La commutation forcée présente un comportement inverse des deux précédentes : son niveau de pollution croît avec la puissance transmise. Il faut noter cependant le rôle favorable, quant aux perturbations de mode différentiel, du circuit d'aide à la mise en conduction. Les multiples circuits d'assistance qui entourent l'interrupteur sont à l'origine de résonances multiples et à haute fréquence (gamme 5 à $25 \mathrm{MHz}$ ). Elles contribuent à maintenir un niveau élevé de bruit.

Dans tous les cas, le ralentissement de la commande d'interrupteur diminue de façon significative les niveaux de pollution, un compromis doit être trouvé avec les pertes par commutation. Cette possibilité est facile à réaliser dans les structures à commutation douce.

A propos de la fréquence de commutation. - Nous avons pu constater que l'augmentation de la fréquence de fonctionnement des convertisseurs conduit à injecter proportionnellement des courants parasites dans les circuits de couplages, provoquant parfois leur résonance et contribuant globalement à accroître le niveau des spectres en haute fréquence.

Il faut distinguer le cas des convertisseurs où la puissance est réglée par variation de fréquence de ceux travaillant à fréquence fixe et rapport cyclique variable. 
Dans le premier cas (structures à commutation douce), il est préférable de choisir, au point nominal, la fréquence de fonctionnement la plus basse compatible avec les contraintes de dimensionnement du convertisseur (Volume, rendement) :

Mode ZVS : Le réglage de puissance du convertisseur évoluant inversement avec la fréquence de découpage est défavorable à l'évolution des régimes parasites, le niveau spectral des bruits augmente lorsque la puissance diminue. Le choix de la fréquence de fonctionnement nominal doit donc être effectué par rapport à sa valeur maximale compatible avec les normes CEM et à son facteur de variation.

Mode ZCS: Le contrôle de puissance s'opérant par réduction de la fréquence est favorable quant à l'aspect fréquentiel de la pollution conduite.

\section{Conclusion.}

Cette étude nous a permis de comprendre les mécanismes de génération et de transmission des perturbations électromagnétiques dans les structures simples que sont les cellules de commutation. Nous avons pu mettre au point une méthode permettant la quantification précise de la perturbation, liée à chaque phase de commutation (amorçage, blocage) et dans les différents modes. Cette démarche permet une caractérisation de l'évolution des régimes perturbants en fonction des paramètres électriques : courant coupé, tension de blocage, fréquence de commutation et vitesse de commande. Cette méthode ouvre par ailleurs des perspectives quant à la quantification de la susceptibilité des circuits environnants.

Il nous semble important de pouvoir simuler le comportement perturbatoire d'un convertisseur, aussi les études doivent se poursuivre dans ce domaine. Nous avons pu mettre au point la méthodologie de simulation qui consiste à localiser un certain nombre de composants parasites dans la structure, ils modifient les formes d'onde théoriques et transmettent les régimes parasites à l'environnement. Nous avons obtenu des résultats intéressants, mais avons aussi montré les limites des simulateurs. La simulation des régimes parasites (pseudo-oscillations dans la gamme 1 à $100 \mathrm{MHz}$ ) nécessite la prise en compte de l'effet de peau en régime impulsionnel dans les câblages et les composants passifs. A l'heure actuelle aucun simulateur de type «circuit» ne peut résoudre ce type de problème. D'autre part, ces phénomènes apparaissent durant les quelques centaines de nanoseconde d'une commutation. Il en résulte que les modèles des interrupteurs et des diodes actuels sont insuffisants dans certains cas (problème de recouvrement de diode, ou de capacité non linéaire), car développés dans l'optique d'une simulation à l'échelle de la période. Ce changement d'échelle doit amener à de nouvelles investigations où les modèles d'interrupteur seront probablement plus complexes. Compte tenu de la prise en compte de nombreux composants parasites et de modèles sophistiqués d'interrupteur, des problèmes de temps de calculs peuvent apparaître, il faut s'interroger sur le solveur le mieux adapté. Un outil CAO en électronique de puissance devra regrouper plusieurs modules permettant la simulation à l'échelle de la période, la modélisation et le calcul des différents composants parasites liés au câblage et à la topologie et enfin la simulation des émissions électromagnétiques à l'échelle de la transition.

Nous avons montré que la réduction des régimes parasites passe par celle des énergies résiduelles, ce qui est facile pour les structures à commutation douce. La minimisation des inductances parasites contribue aussi à cette action, il en est de même des couplages capacitifs parasites. 


\section{Bibliographie}

[1] Lu Bel, Contributıon à l'étude du rayonnement en champ électromagnétique proche des circuits en électronique de puissance, thèse de doctorat de l'Ecole Centrale de Lyon (mai, 1990).

[2] Degauque et Hammelin, Compatibilité électromagnétique (éditions Dunod).

[3] Puzo A., Contribution à l'étude des perturbations rayonnées par les convertisseurs statiques HF, thèse de doctorat de l'Ecole Centrale de Lyon (juin, 1992).

[4] Costa F., Contribution à l'étude des perturbations conduites par les convertisseurs statiques HF, thèse de doctorat de l'université Paris-Sud Orsay (avril, 1992).

[5] Combes, Sautereau, Graffeuil, Composants, dispositifs et circuits actifs en microondes (éditions Dunod).

[6] Hewlet Packard, Designing for EMC. student Workbook n HP 11949A (1989).

[7] Burais N., documentation technique du logiciel FISSURE.

[8] Notice technique des logiciels VALID et SICOS.

[9], [10] Documentation des logiciels DIEL et PHI3D.

[11] Lefebvre S., Costa F., Forest F., Contrôle des gradients de commutation dans les convertisseurs haute fréquence, proceeding du congrès EPF (1992) pp. 5-1/7-5-7/7.

[12] Forest F., Liénard Ph., High performances, medium power DC to DC converter, Proceeding du congrès EPE, vol. III (1989) 1239-1244.

[13] Cheron Y., La commutation douce dans la conversıon statique de l'énergie électrique, Thèse d'état, INP Toulouse (1988).

[14] Meynard T., Cheron Y., Foch H., Generalization of the resonant switch concept : structures and performances, Proceeding du congrès EPE (1987) pp. 239-244. 